\title{
Avoiding order reduction when integrating reaction-diffusion boundary value problems with exponential splitting methods
}

\author{
I. Alonso-Mallo ${ }^{\mathrm{a}}$, B. Cano ${ }^{\mathrm{a}}$, N. Reguera ${ }^{\mathrm{b}, *}$ \\ ${ }^{a}$ IMUVA, Departamento de Matemática Aplicada, Universidad de Valladolid, Facultad de \\ Ciencias, Paseo de Belén 7, 47011 Valladolid, Spain. \\ ${ }^{b}$ IMUVA, Departamento de Matemáticas y Computación, Escuela Politécnica Superior, \\ Universidad de Burgos, Avda. Cantabria, 09006 Burgos, Spain
}

\begin{abstract}
In this paper, we suggest a technique to avoid order reduction in time when integrating reaction-diffusion boundary value problems under non-homogeneous boundary conditions with exponential splitting methods. More precisely, we consider Lie-Trotter and Strang splitting methods and Dirichlet, Neumann and Robin boundary conditions. Beginning from an abstract framework in Banach spaces, a thorough error analysis after full discretization is performed and some numerical results are shown which corroborate the theoretical results.
\end{abstract}

Keywords: Exponential splitting, order reduction, initial boundary value problem.

\section{Introduction}

Exponential methods are very much used in the recent literature when integrating partial differential equations because they integrate the linear and stiff part of the problem in an exact way [22]. Due to the recent and thorough development of Krylov-type methods to calculate exponential-type functions over matrices which are applied over vectors [18], they constitute an effective tool to integrate such problems in a stable way.

In this paper, we will center on exponential splitting methods. More precisely, on the first-order Lie-Trotter and second-order Strang methods when the linear part is integrated in an exact way. The order reduction which turns up with these methods when integrating linear problems with homogeneous boundary conditions was recently studied in [17]. In [1] we have suggested a technique to deal with non-homogeneous boundary conditions in linear problems. That

\footnotetext{
* Corresponding author

Email addresses: isaias@mac.uva.es (I. Alonso-Mallo), bego@mac.uva.es (B. Cano), nreguera@ubu.es (N. Reguera)
} 
technique has some similarities to that suggested in [3] for other exponentialtype methods which also suffer a severe order reduction, which are Lawson ones [2]. With that procedure, we managed to avoid order reduction completely in linear problems.

The aim of the present paper is to generalize that technique to nonlinear reaction-diffusion problems and to prove that order reduction can also be completely avoided. As in [13, 23], the idea is to consider suitable intermediate boundary conditions for the split evolutionary problems. In contrast to those papers, as we consider exponential methods and the boundary values we suggest do not require numerical differentiation, no stability restriction on the grid sizes is needed. Moreover, the class of problems which are treated there is different.

There are other results in the literature concerning the same nonlinear problem as here or a more specific one. For example, in [10], a generalized Strang method is suggested for the specific nonlinear Schrödinger equation. However, in that paper, an abstract formulation of the problem is not given (as it is here), Neumann or Robin type boundary conditions are not considered, parabolic problems for which a summation-by-parts argument can be applied are not included and finally, Lie-Trotter method is not analyzed. On the other hand, in $[15,16]$, a completely different technique is suggested to avoid order reduction with the same methods and nonlinear problems than here, but the analysis for the local and global error is just performed over the time splitting. In such a way, the error coming from the space discretization and the numerical approximation in time of the split problems is not included there. In contrast, in the present paper, that analysis is included and performed under quite general assumptions on the space discretization and time integration of the nonlinear part. For that, we use the maximum norm, which facilitates its applicability to quite general problems. Besides, the error coming from the discretization of the possible Neumann/Robin boundary conditions is also considered. Moreover, for different possible implementations of Strang method, a comparison between the technique in [16] and the one suggested here has been performed in [4] and the technique suggested here turns out to be more efficient (see also [5] where another implementation of the technique in [16] is used).

The paper is structured as follows. Section 2 gives some preliminaries on the abstract setting of the problem, on the assumptions of regularity which are required for the solution to be approximated and on Lie-Trotter and Strang methods. Section 3 describes the technique to avoid order reduction after time integration with Lie-Trotter method and explains how to deal with nonhomogeneous Dirichlet, Robin and Neumann type boundary conditions. Moreover, a thorough local error analysis is given. In Section 4, the same is done for Strang method, for which just order 2 can be obtained in general for the local error. Section 5 states some hypotheses on the space discretization which include some finite-difference schemes, as the ones being used in the numerical experiments. (Similarly, collocation-type methods could be considered.) In Sections 6 and 7, the exact formulas to be implemented after full discretization are described in (38)-(39) for Lie-Trotter and in (47), (49) and (51) for Strang. Moreover, the detailed results on the local and global error after full discretiza- 
tion are given in those sections, although their proofs have been postponed to an appendix. Order reduction is completely avoided with Lie-Trotter and the same happens with Strang method if the bound (45) is satisfied by the discretization of the elliptic problem. Section 8 shows some numerical experiments which corroborate the previous results. Moreover, in two dimensions a double splitting is included considering the results in [1]. Finally, some conclusions are given in Section 9.

\section{Preliminaries}

Let $X$ and $Y$ be Banach spaces with respective norms $\|\cdot\|_{X}$ and $\|\cdot\|_{Y}$, and let $A: D(A) \rightarrow X$ and $\partial: X \rightarrow Y$ be linear operators. Our goal is to study full discretizations, by using as time integrators Lie-Trotter and Strang exponential methods, of the nonlinear abstract non homogeneous initial boundary value problem

$$
\begin{aligned}
u^{\prime}(t) & =A u(t)+f(t, u(t)), \quad 0 \leq t \leq T, \\
u(0) & =u_{0} \in X, \\
\partial u(t) & =g(t) \in Y, \quad 0 \leq t \leq T,
\end{aligned}
$$

where the functions $f:[0, T] \times X \rightarrow X$ (in general nonlinear) and $g:[0, T] \rightarrow Y$ are regular enough.

The abstract setting (1) permits to cover a wide range of nonlinear evolutionary problems governed by partial differential equations. We use the following hypotheses, which are closely related to the ones in [19], where the Strang splitting applied to a similar abstract problem with homogeneous boundary conditions is studied. In our case, we add suitable hypotheses in a such way that we are able to consider non homogeneous boundary values (cf. [6, 24]).

(A1) The boundary operator $\partial: D(A) \subset X \rightarrow Y$ is onto.

(A2) $\operatorname{Ker}(\partial)$ is dense in $X$ and $A_{0}: D\left(A_{0}\right)=\operatorname{ker}(\partial) \subset X \rightarrow X$, the restriction of $A$ to $\operatorname{Ker}(\partial)$, is the infinitesimal generator of a $C_{0}$ - semigroup $\left\{e^{t A_{0}}\right\}_{t \geq 0}$ in $X$, which type $\omega$ is assumed to be negative.

(A3) If $z \in \mathbb{C}$ satisfies $\Re(z)>\omega$ and $v \in Y$, then the steady state problem

$$
\begin{aligned}
& A x=z x, \\
& \partial x=v,
\end{aligned}
$$

possesses a unique solution denoted by $x=K(z) v$. Moreover, the linear operator $K(z): Y \rightarrow D(A)$ satisfies

$$
\|K(z) v\|_{X} \leq C\|v\|_{Y}
$$

where the constant $C$ holds for any $z$ such that $\operatorname{Re}(z) \geq \omega_{0}>\omega$.

(A4) The nonlinear source $f$ belongs to $C^{1}([0, T] \times X, X)$.

(A5) The solution $u$ of (1) satisfies $u \in C^{2}([0, T], X), u(t) \in D\left(A^{2}\right)$ for all $t \in[0, T]$ and $A u, A^{2} u \in C^{1}([0, T], X)$. 
(A6) $f(t, u(t)) \in D(A)$ for all $t \in[0, T]$, and $A f(\cdot, u(\cdot)) \in C([0, T], X)$.

In the remaining of the paper, we always suppose that (A1)-(A5) are satisfied. Assumption (A6) will just be required for the results on Strang method. Moreover, we notice that we will also assume more regularity on $u$ and $f$ for some of the results which correspond to the full discretization. (See Theorems 12, 14 and 15.)

Remark 1. From (A4), we deduce that $f: D(f) \subset[0, T] \times X \rightarrow X$ is locally Lipschitz continuous in $u$, uniformly in $t \in[0, T]$, with respect to the norm in $X$, that is,

$$
\|f(t, v)-f(t, u)\|_{X} \leq L(c)\|v-u\|_{X},
$$

for $(t, u),(t, v) \in D(f)$ with $\|u\|_{X},\|v\|_{X} \leq c$.

In order to define the Lie-Trotter and Strang splitting methods, we need to solve the nonlinear evolution equation

$$
\begin{aligned}
v^{\prime}(t) & =f(\tau+t, v(t)), \\
v(0) & =v_{0},
\end{aligned}
$$

for several initial values $v_{0}$ and times $\tau>0$. From (5), problem (6) has a unique solution, which is well defined for sufficiently small times (see Theorem 1.8.1 in [12]).

Remark 2. When the problem (1) is linear, that is, when $f(t, \cdot) \equiv h(t)$, the results in [6, 24] show that, with the hypotheses (A1)-(A3), the problem

$$
\begin{aligned}
u^{\prime}(t) & =A u(t)+h(t), \quad 0 \leq t \leq T, \\
u(0) & =u_{0} \in X, \\
\partial u(t) & =g(t) \in Y, \quad 0 \leq t \leq T,
\end{aligned}
$$

is well posed and the solution depends continuously on data $u_{0}, h$, and $g$.

In order to define the time integrators which are used in this paper, we will consider initial boundary value problems which can be written as

$$
\begin{aligned}
u^{\prime}(s) & =A u(s), \\
u(0) & =u_{0}, \\
\partial u(s) & =v_{0}+v_{1} s,
\end{aligned}
$$

where $u_{0} \in X$ and $v_{0}, v_{1} \in Y$.

Assuming that $u_{0} \in D(A)$ and $\partial u_{0}=v_{0}$, the solution of (8) is given by (see e.g. [1])

$$
u(t)=e^{t A_{0}}\left(u_{0}-K(0) v_{0}\right)+K(0)\left(v_{0}+v_{1} t\right)-\int_{0}^{t} e^{s A_{0}} K(0) v_{1} d s .
$$

Notice that (9) is well defined for any $u_{0} \in X$ and $v_{0}, v_{1} \in Y$; therefore, it may be considered as a generalized solution of (8) even when $\partial u_{0} \neq v_{0}$ or $u_{0} \notin D(A)$. We will use this fact in order to establish the time integrator method in the following section. 
Remark 3. From hypotheses (A1)-(A4), problem (1) with homogeneous boundary conditions has a unique classical solution for small enough time intervals (see Theorem 6.1.5 in [25]).

Regarding the nonhomogeneous case, we can assume that the boundary function $g:[0, T] \rightarrow Y$ satisfies $g \in C^{1}([0, T], Y)$ and we can look for a solution of (1) given by:

$$
u(t)=v(t)+K(z) g(t), \quad t \geq 0,
$$

for some fixed $\Re(z)>\omega$. Then, $v$ is the solution of an IBVP with vanishing boundary values similar to the one in [25] and the well-posedness for the case of nonhomogeneous boundary values is a direct consequence if we take the abstract theory for initial boundary value problems in [6, 24] into account.

However, condition (A4) may be very strong. When $X$ is a function space with a norm $L^{p}, 1 \leq p<+\infty$, and $f$ is an operator given by,

$$
u \rightarrow f(u)=\phi \circ u
$$

with $\phi: \mathbb{C} \rightarrow \mathbb{C},(5)$ implies that $\phi$ is globally Lipschitz in $\mathbb{C}$. This objection disappears by considering the supremum norm, which is used in our numerical examples, where the nonlinear source is given by

$$
u \rightarrow f(t, u)=\phi \circ u+h(t)
$$

with $h:[0, T] \rightarrow X$, that is, $f$ is the sum of an operator like (10) and a linear term. In this way, problem (1) is well posed whenever $\phi$ and $h$ are $C^{1}$.

Remark 4. If we suppose that $A_{0}$ is the infinitesimal generator of an analytic semigroup, we can consider, for $\theta \in(0,1)$ a new norm given by $\|u\|_{\theta}=\|(\omega I-$ $\left.A_{0}\right)^{\theta} u \|, \omega>0$, when $u \in X_{\theta}=D\left(\left(\omega I-A_{0}\right)^{\theta}\right)$. In this case, if $f$ satisfies $a$ local Lipschitz condition in $u$ with this new norm, it is possible to obtain the well posedness of problem (1) even when $X$ is a function space with a norm $L^{p}$, $1 \leq p<+\infty$ (see [20]). However, this approach is not enough for our purposes since we also need to solve the nonlinear evolution equation (6).

Example. Let $\Omega \subset \mathbb{R}^{n}$ be a bounded domain with Lipschitz boundary. Then, there exists a unique solution $h \in C(\bar{\Omega})$ of the problem

$$
\begin{aligned}
\Delta h & =0 \text { in } D(\Omega)^{\prime}, \\
\left.h\right|_{\partial \Omega} & =\varphi \in C(\partial \Omega),
\end{aligned}
$$

where $D(\Omega)^{\prime}$ denotes the space of distributions. We also remark that it can be proved that $h \in C^{\infty}(\Omega)$.

We take $X=C(\bar{\Omega})$ with the supremum norm and we consider the operator $A_{0}$ defined on $X$ by

$$
\begin{aligned}
D\left(A_{0}\right) & =\left\{u \in C_{0}(\Omega): \Delta u \in X\right\} \\
A_{0} u & =\Delta u \in D(\Omega)^{\prime}
\end{aligned}
$$


where $C_{0}(\Omega)=\left\{u \in X:\left.u\right|_{\partial \Omega}=0\right\}$. Then, the operator $A_{0}$ generates a bounded holomorphic semigroup $e^{t A_{0}}$ on $X$ ([7], Section 2.4). We denote $\omega<0$ the type of this semigroup.

Now, we take $Y=C(\partial \Omega)$ and we define the linear operator

$$
\begin{aligned}
K: Y & \rightarrow K(Y) \subset X \\
\varphi & \rightarrow K(\varphi)=h,
\end{aligned}
$$

where $h$ is the solution of (12). Then, we can define the (dense) subspace

$$
D(A)=D\left(A_{0}\right) \oplus K(Y),
$$

the extension of the operator $A_{0}$,

$$
A: D(A) \subset X \rightarrow X
$$

by means of $A u=\Delta u$ for each $u \in D(A)$, and the boundary operator

$$
\begin{aligned}
\partial: D(A) \subset X & \rightarrow Y, \\
u & \rightarrow \partial u=\left.u\right|_{\partial \Omega} .
\end{aligned}
$$

Finally, if $z \in \mathbb{C}$ satisfies $\Re(z)>\omega$, we define

$$
K(z)=\left(-A_{0}\right)\left(z-A_{0}\right)^{-1} K=K-z\left(z-A_{0}\right)^{-1} K,
$$

which satisfies $A K(z)=A K-z A_{0}\left(z-A_{0}\right)^{-1} K=-z A_{0}\left(z-A_{0}\right)^{-1} K=z K(z)$.

Therefore, hypotheses (A1),(A2), and (A3) are satisfied.

We remark that the restriction to $A=\Delta$ is only made for simplicity of presentation and more general elliptic operators can be considered (see [8]).

Because of hypothesis (A2), $\left\{\varphi_{j}\left(t A_{0}\right)\right\}_{j=1}^{3}$ are bounded operators for $t>0$, where $\left\{\varphi_{j}\right\}$ are the standard functions which are used in exponential methods $[22]$ and which are defined by

$$
\varphi_{j}\left(t A_{0}\right)=\frac{1}{t^{j}} \int_{0}^{t} e^{(t-\tau) A_{0}} \frac{\tau^{j-1}}{(j-1) !} d \tau, \quad j \geq 1 .
$$

It is well-known that they can be calculated in a recursive way through the formulas

$$
\varphi_{j+1}(z)=\frac{\varphi_{j}(z)-1 / j !}{z}, z \neq 0, \quad \varphi_{j+1}(0)=\frac{1}{(j+1) !}, \quad \varphi_{0}(z)=e^{z} .
$$

For the time integration, we will center on exponential Lie-Trotter and Strang methods which, applied to a finite-dimensional nonlinear problem like

$$
U^{\prime}(t)=M U(t)+F(t, U(t)),
$$

where $M$ is a matrix, are described by the following formulas at each step

$$
\begin{aligned}
U_{n+1} & =\Psi_{k}^{F, t_{n}}\left(e^{k M} U_{n}\right), \\
U_{n+1} & =\Psi_{\frac{k}{2}}^{F, t_{n}+\frac{k}{2}}\left(e^{k M} \Psi_{\frac{k}{2}}^{F, t_{n}}\left(U_{n}\right)\right),
\end{aligned}
$$


where $k>0$ is the time stepsize and $\Psi_{k}^{F, t_{n}}(U)$ and $\Psi_{k}^{F, t_{n}+\frac{k}{2}}(U)$ are the results of applying a certain $p$ th-order numerical method $(p \geq 1)$ to the following nonlinear differential problems:

$$
U^{\prime}(s)=F\left(t_{n}+s, U(s)\right), \quad U^{\prime}(s)=F\left(t_{n}+\frac{k}{2}+s, U(s)\right),
$$

with initial condition $U(0)=U$ and $t_{n}=n k$ for $n \geq 0$.

\section{Time semidiscretization: exponential Lie-Trotter splitting}

In this section, we give the technique to generalize Lie-Trotter exponential method, so that time order reduction is avoided even with non-vanishing and time-dependent boundary conditions. Besides, we prove the full-order of the local error of the time semidiscretization.

\subsection{Description of the technique}

Whenever $M$ is a matrix, $e^{s M} V$ is the solution at $t=s$ of

$$
\begin{aligned}
\dot{U}(t) & =M U(t), \\
U(0) & =V .
\end{aligned}
$$

More generally, matrix $M$ can be substituted by the infinitesimal generator $A_{0}$ of a $C_{0}$-semigroup in a certain Banach space $X$. Then, the corresponding semigroup is denoted by $e^{s A_{0}}$ and $e^{s A_{0}} v$, for $v \in D\left(A_{0}\right) \subset X$, is the solution of the corresponding abstract differential problem

$$
\begin{aligned}
\dot{u}(t) & =A_{0} u(t), \\
u(0) & =v .
\end{aligned}
$$

When $A_{0}$ is a linear (unbounded) operator associated to a differential operator defined on $\Omega \subset \mathbb{R}^{n}$, its domain $D\left(A_{0}\right)$ is formed by functions for which certain boundary operator vanishes on the boundary of $\Omega$ (see Example in Section 2).

Since we are interested in problems with nonvanishing boundary conditions, as those in (1), we replace the exponential matrices or semigroups with the solution of differential problems where the boundary values must be specified in a clever way. More precisely, we suggest to advance a stepsize from $u_{n}$ in the following way. Firstly, we consider the solution of

$$
\begin{aligned}
v_{n}^{\prime}(s) & =A v_{n}(s), \\
v_{n}(0) & =u_{n}, \\
\partial v_{n}(s) & =\partial \hat{v}_{n}(s),
\end{aligned}
$$

where

$$
\hat{v}_{n}(s)=u\left(t_{n}\right)+s A u\left(t_{n}\right) .
$$


Then, we consider the problem

$$
\begin{aligned}
& w_{n}^{\prime}(s)=f\left(t_{n}+s, w_{n}(s)\right), \\
& w_{n}(0)=v_{n}(k),
\end{aligned}
$$

and $u^{n+1}$ is obtained advancing a time step $k \geq 0$ by means of a numerical integrator of order $p \geq 1$. That is,

$$
u^{n+1}=\Psi_{k}^{f, t_{n}}\left(v_{n}(k)\right) .
$$

Notice that we could have also started by integrating the nonlinear part of the equation and then the linear and stiff one. However, that would have led to a slightly more complicated expression for the boundary in the linear part.

\subsection{Calculation of the required boundaries}

In order to calculate $\partial \hat{v}_{n}(s)$, apart from $\partial u\left(t_{n}\right)=g\left(t_{n}\right)$, we also need $\partial A u\left(t_{n}\right)$, for which we can use from (1),

$$
\partial A u\left(t_{n}\right)=\partial u^{\prime}\left(t_{n}\right)-\partial f\left(t_{n}, u\left(t_{n}\right)\right)=g^{\prime}\left(t_{n}\right)-\partial f\left(t_{n}, u\left(t_{n}\right)\right) .
$$

When the operator $\partial$ corresponds to a Dirichlet boundary condition and the nonlinear term is given by (11),

$$
\partial f\left(t_{n}, u\left(t_{n}\right)\right)=\phi \circ g\left(t_{n}\right)+\partial h\left(t_{n}\right),
$$

and $\partial \hat{v}_{n}(s)$ is exactly calculated from the given data. However, when $\partial$ corresponds to a Robin or Neumann boundary condition, $\partial A u\left(t_{n}\right)$ can only be calculated in an approximated way. For that, we write the boundary condition as

$$
\partial u=\left.\alpha u\right|_{\partial \Omega}+\left.\beta \partial_{n} u\right|_{\partial \Omega}=g, \quad \beta \neq 0,
$$

with $\partial \Omega$ the boundary (or some part of it) of some domain $\Omega$ and $\partial_{n}$ the normal derivative to that boundary. Then, when $f$ is again like in (11), it can be used that

$\partial f\left(t_{n}, u\left(t_{n}\right)\right)=\alpha\left[\phi\left(\left.u\left(t_{n}\right)\right|_{\partial \Omega}\right)+\left.h\left(t_{n}\right)\right|_{\partial \Omega}\right]+\beta\left[\left.\phi^{\prime}\left(\left.u\left(t_{n}\right)\right|_{\partial \Omega}\right) \partial_{n} u\left(t_{n}\right)\right|_{\partial \Omega}+\left.\partial_{n} h\left(t_{n}\right)\right|_{\partial \Omega}\right]$.

In this expression, $\left.u\left(t_{n}\right)\right|_{\partial \Omega}$ can be substituted by the numerical approximation at the previous step and $\left.\partial_{n} u\left(t_{n}\right)\right|_{\partial \Omega}$ by the result of applying the following formula which comes from (23)

$$
\left.\partial_{n} u\right|_{\partial \Omega}=\frac{g\left(t_{n}\right)-\left.\alpha u\left(t_{n}\right)\right|_{\partial \Omega}}{\beta} .
$$




\subsection{Local error of the time semidiscretization}

In order to study the local error, we consider the value which is obtained in (22) starting from $u\left(t_{n}\right)$ in (19). Then, we obtain

$$
\bar{u}_{n+1}=\Psi_{k}^{f, t_{n}}\left(\bar{v}_{n}(k)\right),
$$

where $\bar{v}_{n}(s)$ is the solution of

$$
\begin{aligned}
\bar{v}_{n}^{\prime}(s) & =A \bar{v}_{n}(s), \\
\bar{v}_{n}(0) & =u\left(t_{n}\right), \\
\partial \bar{v}_{n}(s) & =\partial \hat{v}_{n}(s) .
\end{aligned}
$$

with $\hat{v}_{n}(s)$ that in $(20)$. The following theorem, which proof is given in the appendix, then follows:

Theorem 5. Let us assume hypotheses (A1)-(A5) and that the numerical integrator $\Psi_{k}$ integrates (21) with order $p \geq 1$ in $X$. Then, when integrating (1) with Lie-Trotter method using the technique (19)-(22), the local error satisfies

$$
\rho_{n+1} \equiv \bar{u}_{n+1}-u\left(t_{n+1}\right)=O\left(k^{2}\right) .
$$

\section{Time semidiscretization: exponential Strang splitting}

With the same idea as in Section 3, we describe now how to generalize Strang exponential method in order to fight against order reduction in time. Instead of achieving order 3 for the local error (as when integrating non-stiff ODEs), we will just achieve order 2 for it. This is due to the fact that we want to guarantee that the boundary of the intermediate evolutionary partial differential equation problem can be calculated without resorting to numerical differentiation. However, as we will see in Sections 7.3 and 8, that will mean in practice no order reduction for the global error because of a summation-by-parts argument.

Notice that, instead of starting with the integration of the linear part, as with Lie-Trotter method, we start with that of the nonlinear and smooth one. This is because, in such a way, just one stiff differential evolutionary problem per step arises for which we must suggest a boundary.

\subsection{Description of the technique}

For the time integration of (1), we firstly consider the problem

$$
\begin{aligned}
& v_{n}^{\prime}(s)=f\left(t_{n}+s, v_{n}(s)\right), \\
& v_{n}(0)=u_{n},
\end{aligned}
$$

and denote by $\Psi_{\frac{k}{2}}^{f, t_{n}}\left(u_{n}\right)$ the numerical approximation of this problem after time $k / 2$. Secondly, we consider

$$
\begin{aligned}
w_{n}^{\prime}(s) & =A w_{n}(s), \\
w_{n}(0) & =\Psi_{k}^{f, t_{n}}\left(u_{n}\right), \\
\partial w_{n}(s) & =\partial \widehat{w}_{n}(s),
\end{aligned}
$$


where

$$
\widehat{w}_{n}(s)=u\left(t_{n}\right)+\frac{k}{2} f\left(t_{n}, u\left(t_{n}\right)\right)+s A u\left(t_{n}\right),
$$

which comes from approximating $v_{n}\left(\frac{k}{2}\right)+s A v_{n}\left(\frac{k}{2}\right)$. Thirdly, by considering

$$
\begin{aligned}
& z_{n}^{\prime}(s)=f\left(t_{n}+\frac{k}{2}+s, z_{n}(s)\right), \\
& z_{n}(0)=w_{n}(k),
\end{aligned}
$$

and advancing $k / 2$ with the numerical integrator, we obtain

$$
u_{n+1}=\Psi_{\frac{k}{2}}^{f, t_{n}+\frac{k}{2}}\left(w_{n}(k)\right) .
$$

Notice that the boundary values in (26) can be exactly or approximately calculated in terms of data under the same considerations of Subsection 3.2.

\subsection{Local error of the time semidiscretization}

In order to study the local error, we consider the value $\bar{u}_{n+1}$ which is obtained in (29) starting from $u_{n}=u\left(t_{n}\right)$ in (25). We then have the following result, which proof is given in the appendix.

Theorem 6. Let us assume that hypotheses (A1)-(A6) are satisfied, and that the numerical integrator $\Psi_{k}$ integrates (25) and (28) with order $p \geq 1$ in $X$. Then, when integrating (1) with Strang method using the technique (25)-(28), the local error satisfies

$$
\rho_{n+1} \equiv \bar{u}_{n+1}-u\left(t_{n+1}\right)=O\left(k^{2}\right) .
$$

Moreover, assuming a bit more regularity of the functions $u$ and $f$ and a bit more accuracy of the time numerical integrator for the nonlinear part, we have the following result:

Theorem 7. Whenever, apart from hypotheses (A1)-(A6), $u \in C^{3}([0, T], X)$ and $f \in C^{2}([0, T] \times X, X)$, when integrating (1) with Strang method using the technique (25)-(29) with a numerical integrator $\Psi_{k}$ which is of order $p \geq 2$ for problems (25) and (28), the local error satisfies

$$
A_{0}^{-1} \rho_{n+1}=O\left(k^{3}\right) \text {. }
$$

\section{Spatial discretization}

Following the example in Section 2, from now on we take $X=C(\bar{\Omega})$ with the maximum norm and we consider a certain grid $\Omega_{h}$ (of $\Omega$ ) over which the approximated numerical solution will be defined. In this way, this numerical approximation belongs to $\mathbb{C}^{N}$, where $N$ is the number of nodes in the grid, endowed with the the maximum norm $\left\|u_{h}\right\|_{h}=\left\|\left[u_{1}, \ldots, u_{N}\right]^{T}\right\|_{h}=\max _{1 \leq i \leq N}\left|u_{i}\right|$. 
Notice that, usually, when considering Dirichlet boundary conditions, nodes on the boundary are not considered while, when using Neumann or Robin boundary conditions, the nodes on the boundary are taken into account.

In that sense, we consider the projection operator

$$
P_{h}: X \rightarrow \mathbb{C}^{N}
$$

which takes a function to its values over the grid $\Omega_{h}$. On the other hand, the operator $A$, when applied over functions which satisfy a certain condition on the boundary $\partial u=g$, is discretized by means of an operator

$$
A_{h, g}: \mathbb{C}^{N} \rightarrow \mathbb{C}^{N}
$$

which takes the boundary values into account. More precisely,

$$
A_{h, g} U_{h}=A_{h, 0} U_{h}+C_{h} g,
$$

where $A_{h, 0}$ is the matrix which discretizes $A_{0}$ and $C_{h}: Y \rightarrow \mathbb{C}^{N}$ is another operator, which is the one which contains the information on the boundary.

We also assume that the source function $f$ has also sense as function from $[0, T] \times \mathbb{C}^{N}$ on $\mathbb{C}^{N}$ and, for each $t \in[0, T]$ and $u \in X$,

$$
P_{h} f(t, u)=f\left(t, P_{h} u\right) .
$$

This fact is obvious when $f$ is given by (11). By using this, the following semidiscrete problem arises after discretising (1) in space,

$$
\begin{aligned}
U_{h}^{\prime}(t) & =A_{h, 0} U_{h}(t)+C_{h} g(t)+f\left(t, U_{h}(t)\right), \\
U_{h}(0) & =P_{h} u(0),
\end{aligned}
$$

The subsequent analysis is carried out under the following hypotheses:

(H1) The matrix $A_{h, 0}$ satisfies

(a) $\left\|e^{t A_{h, 0}}\right\|_{h} \leq C, 0 \leq t \leq T$, for some constant $C$ which does not depend on $h$ either on $t$,

(b) $A_{h, 0}$ is invertible and $\left\|A_{h, 0}^{-1}\right\|_{h} \leq C^{\prime}$ for some constant $C^{\prime}$ which does not depend on $h$,

where $\|\cdot\|_{h}$ is the norm operator obtained from the maximum norm in $\mathbb{C}^{N}$.

(H2) We define the elliptic projection $R_{h}: D(A) \rightarrow \mathbb{C}^{N}$ as the solution of

$$
A_{h, 0} R_{h} u+C_{h} \partial u=P_{h} A u .
$$

We assume that there exists a subspace $Z \subset D(A)$, such that, for $u \in Z$,

(a) $A_{0}^{-1} u \in Z$ and $e^{t A_{0}} u \in Z$, for $t \geq 0$.

(b) for some $\varepsilon_{h}$ and $\eta_{h}$ which are both small with $h$,

$$
\left\|A_{h, 0}\left(P_{h} u-R_{h} u\right)\right\|_{h} \leq \varepsilon_{h}\|u\|_{Z}, \quad\left\|P_{h} u-R_{h} u\right\|_{h} \leq \eta_{h}\|u\|_{Z} .
$$

(Although obviously, because of (H1), $\eta_{h}$ could be taken as $C \varepsilon_{h}$, for some discretizations $\eta_{h}$ can decrease more quickly with $h$ than $\varepsilon_{h}$ and that leads to better error bounds in the following section.) 
We also assume that a discrete maximum principle applies for this discretization, i.e.

(c) $\left\|A_{h, 0}^{-1} C_{h}\right\|_{h} \leq C^{\prime \prime}$ for some constant $C^{\prime \prime}$ which does not depend on $h$. This resembles the continuous maximum principle which is satisfied because of (4) when $z=0$.

(H3) The nonlinear source $f$ belongs to $C^{1}\left([0, T] \times \mathbb{C}^{N}, \mathbb{C}^{N}\right)$ and the derivative with respect to the variable $u_{h}$ is uniformly bounded in a neighbourhood of the solution where the numerical approximation stays.

Notice that, from (H3), the non linear term $f$ satisfies, for some Lipschitz constant $L$ independent of $t \in[0, T]$ and the maximum norm,

$$
\left\|f\left(t, v_{h}\right)-f\left(t, u_{h}\right)\right\|_{h} \leq L\left\|v_{h}-u_{h}\right\|_{h},
$$

when $u_{h}, v_{h}$ belong to a compact set. In particular, we will be interested in considering as this set a neighborhood of the exact solution where the numerical approximation stays.

\section{Full discretization: exponential Lie-Trotter splitting}

6.1. Final formula for the implementation

We apply the above space discretization to the evolutionary problems (19) and (21) and we obtain $V_{h, n}(s), W_{h, n}(s)$ in $\mathbb{C}^{N}$ as the solutions of

$$
\begin{aligned}
& V_{h, n}^{\prime}(s)=A_{h, 0} V_{h, n}(s)+C_{h} \partial \hat{v}_{n}(s), \\
& V_{h, n}(0)=U_{h}^{n},
\end{aligned}
$$

where $\hat{v}_{n}(s)$ is that in (20), $U_{h}^{n} \in \mathbb{C}^{N}$ is the numerical solution in the interior of the domain after full discretization at $n$ steps, and

$$
\begin{aligned}
& W_{h, n}^{\prime}(s)=f\left(t_{n}+s, W_{h, n}(s)\right), \\
& W_{h, n}(0)=V_{h, n}(k) .
\end{aligned}
$$

By using the variations of constants formula and the definition of the functions $\varphi_{1}$ and $\varphi_{2}$ in (13),

$$
\begin{aligned}
V_{h, n}(k)= & e^{k A_{h, 0}} U_{h}^{n}+\int_{0}^{k} e^{(k-s) A_{h, 0}}\left[C_{h} \partial\left[u\left(t_{n}\right)+s A u\left(t_{n}\right)\right]\right] d s \\
= & e^{k A_{h, 0}} U_{h}^{n}+k \varphi_{1}\left(k A_{h, 0}\right) C_{h} g\left(t_{n}\right) \\
& +k^{2} \varphi_{2}\left(k A_{h, 0}\right) C_{h}\left(g^{\prime}\left(t_{n}\right)-\partial f\left(t_{n}, u\left(t_{n}\right)\right)\right),
\end{aligned}
$$

and the numerical solution at step $n+1$ is therefore given by

$$
U_{h}^{n+1}=\Psi_{k}^{f, t_{n}}\left(V_{h, n}(k)\right),
$$

where $\Psi_{k}^{f, t_{n}}$ stands for the previously mentioned numerical integrator applied to $(37)$.

Moreover, we will take, as initial condition,

$$
U_{h}^{0}=P_{h} u(0) .
$$


Remark 8. Notice that, when

$$
\partial u\left(t_{n}\right)=\partial A u\left(t_{n}\right)=0,
$$

it is also deduced from (1) that $\partial f\left(t_{n}, u\left(t_{n}\right)\right)=0$. Therefore, the formulas (38)(39) just reduce to the standard time integration with Lie-Trotter method of the differential system which arises after discretizing (1) directly in space (see (32)):

$$
U_{h}^{\prime}(t)=A_{h, 0} U_{h}(t)+f\left(t, U_{h}(t)\right) .
$$

Because of that, with the results which follow, we will be implicitly proving that there is no order reduction in the local error with the standard Lie-Trotter method under these assumptions.

Remark 9. We notice that, when $k$ is fixed, $e^{k A_{h, 0}}$ and $\varphi_{j}\left(k A_{h, 0}\right)$ could be calculated once and for all at the very beginning. Besides, as better explained in the numerical experiments, $C_{h}$ will be represented by a matrix of dimension $O\left(\hat{N}^{d}\right) \times O\left(\hat{N}^{d-1}\right)$ where $d$ is the dimension of the problem and $\hat{N}$ the number of grid points in each direction. Then, $\varphi_{j}\left(k A_{h, 0}\right) C_{h}(j=1,2)$ will be represented by matrices of the same order and therefore the computational cost of calculating the product of those matrices times the information on the boundary values is $O\left(\hat{N}^{2 d-1}\right)$, which is negligible compared with $O\left(\hat{N}^{2 d}\right)$, which corresponds to the calculation of the product of $e^{k A_{h, 0}}$ times a vector of size $O\left(\hat{N}^{d}\right)$. On the other hand, for fixed and variable timestepsize $k$, Krylov techniques can also be used to calculate the terms in (38) without explicitly calculating $e^{k A_{h, 0}}, \varphi_{j}\left(k A_{h, 0}\right)$ $(j=1,2)$. As suggested in [18], it seems in principle cheaper to calculate the terms containing the $\varphi_{j}$-functions than those corresponding to the exponentials.

\subsection{Local errors}

In order to define the local error, we consider

$$
\bar{U}_{h}^{n+1}=\Psi_{k}^{f, t_{n}}\left(\bar{V}_{h, n}(k)\right),
$$

where $\bar{V}_{h, n}(s)$ is the solution of

$$
\begin{aligned}
\bar{V}_{h, n}^{\prime}(s) & =A_{h, 0} \bar{V}_{h, n}(s)+C_{h} \partial \hat{v}_{n}(s), \\
\bar{V}_{h, n}(0) & =P_{h} u\left(t_{n}\right) .
\end{aligned}
$$

with $\hat{v}_{n}(s)$ that in (20). We now define the local error at $t=t_{n}$ as

$$
\rho_{h, n}=P_{h} u\left(t_{n}\right)-\bar{U}_{h}^{n},
$$

and study its behaviour in the following theorem, whose proof is given in the appendix. 
Theorem 10. Let us assume hypotheses (A1)-(A5), that $\Psi_{k}$ integrates (37) with order $p \geq 1$ and (H1)-(H3). Then, when integrating (1) with Lie-Trotter method as described in (38)-(39), whenever u satisfies

$$
u, A u, A^{2} u \in C([0, T], Z),
$$

for the space $Z$ in (H3), the local error after full discretization satisfies

$$
\rho_{h, n+1}=O\left(k \varepsilon_{h}+k^{2}\right), \quad A_{h, 0}^{-1} \rho_{h, n+1}=O\left(k \eta_{h}+k^{2}\right) .
$$

where $\varepsilon_{h}$ and $\eta_{h}$ are those in (34).

\subsection{Global errors}

We now study the global errors at $t=t_{n}$, which are given by

$$
e_{h, n}=P_{h} u\left(t_{n}\right)-U_{h}^{n} .
$$

We have the following result:

Theorem 11. Under the same assumptions of Theorem 10, and assuming also that $\partial f\left(t_{n}, u\left(t_{n}\right)\right)$ can be calculated exactly from data according to Subsection 3.2, the global error which turns up when integrating (1) through formulas (38)(40) satisfies

$$
e_{h, n}=O\left(k+\varepsilon_{h}\right),
$$

where $\varepsilon_{h}$ is that in (34).

Another finer result is the following, which will be very useful for nonDirichlet boundary conditions, when $\partial f\left(t_{n}, u\left(t_{n}\right)\right.$ cannot be calculated exactly but following Subsection 3.2.

Theorem 12. Let us assume the same hypotheses of Theorem 10, that u belongs to $C^{3}([0, T], X)$ and $A f(\cdot, u(\cdot)), f_{t}(\cdot, u(\cdot)), f_{u}(\cdot, u(\cdot))$ to $C^{1}([0, T], X)$, that $f$ is like in (11) with $\phi \in C^{2}(\mathbb{C}, \mathbb{C})$ and and also that there exists a constant $C$, independent of $h$, such that

$$
\left\|k A_{h, 0} \sum_{r=1}^{n-1} e^{r k A_{h, 0}}\right\|_{h} \leq C, \quad 0 \leq n k \leq T .
$$

Then, the global error satisfies

$$
e_{h, n}=O\left(k+\eta_{h}+k \varepsilon_{h}\right),
$$

where $\eta_{h}$ and $\varepsilon_{h}$ are those in (34).

Notice that the bound (45) has been proved in [21] for analytic semigroups, covering the case in which the linear operator in (1) corresponds to that of a parabolic problem. 


\section{Full discretization: exponential Strang splitting}

\subsection{Final formula for the implementation}

Firstly, we apply the space discretization in Section 5 to the evolutionary problem (25) and we obtain $V_{h, n}(s) \in \mathbb{C}^{N}$ as the solution of

$$
\begin{aligned}
& V_{h, n}^{\prime}(s)=f\left(t_{n}+s, V_{h, n}(s)\right), \\
& V_{h, n}(0)=U_{h}^{n} .
\end{aligned}
$$

We will have to use the numerical integrator $\Psi$ in order to approximate the solution of this problem. Then, we define

$$
V_{h}^{n}=\Psi_{\frac{k}{2}}^{f, t_{n}}\left(U_{h}^{n}\right)
$$

As a second step, discretizing (26), we consider $W_{h, n}(s) \in \mathbb{C}^{N}$ as the solution of

$$
\begin{aligned}
& W_{h, n}^{\prime}(s)=A_{h, 0} W_{h, n}(s)+C_{h} \partial \hat{w}_{n}(s), \\
& W_{h, n}(0)=V_{h}^{n} .
\end{aligned}
$$

where $\hat{w}_{n}(s)$ is that in (27). By using the variations of constants formula and the definition of the functions $\varphi_{1}$ and $\varphi_{2}$ in (13), we can solve this problem exactly and we get

$$
\begin{aligned}
W_{h, n}(k)= & e^{k A_{h, 0}} V_{h}^{n}+\int_{0}^{k} e^{(k-s) A_{h, 0}} C_{h} \partial \hat{w}_{n}(s) d s \\
= & e^{k A_{h, 0}} V_{h}^{n}+k \varphi_{1}\left(k A_{h, 0}\right) C_{h}\left[g\left(t_{n}\right)+\frac{k}{2} \partial f\left(t_{n}, u\left(t_{n}\right)\right)\right] \\
& +k^{2} \varphi_{2}\left(k A_{h, 0}\right) C_{h}\left[g^{\prime}\left(t_{n}\right)-\partial f\left(t_{n}, u\left(t_{n}\right)\right]\right.
\end{aligned}
$$

Finally, from (28), we consider $Z_{h, n}(s) \in \mathbb{C}^{N}$ as the solution of

$$
\begin{aligned}
& Z_{h, n}^{\prime}(s)=f\left(t_{n}+\frac{k}{2}+s, Z_{h, n}(s)\right) \\
& Z_{h, n}(0)=W_{h, n}(k)
\end{aligned}
$$

and, numerically integrating this problem, we obtain

$$
U_{h}^{n+1}=\Psi_{\frac{k}{2}}^{f, t_{n}+\frac{k}{2}}\left(W_{h, n}(k)\right) .
$$

Remark 13. Similar comments to those in Remark 8 apply here. Therefore, when $\partial u(t)=\partial A u(t)=0$, with the results which follow we will be implicitly proving that the standard discretization with Strang method gives to rise to order 2 for the local error. 


\subsection{Local error}

In order to define the local error, we consider

$$
\bar{U}_{h}^{n+1}=\Psi_{\frac{k}{2}}^{f, t_{n}+\frac{k}{2}}\left(\bar{W}_{h, n}(k)\right),
$$

where $\bar{W}_{h, n}(s)$ is the solution of

$$
\begin{aligned}
& \bar{W}_{h, n}^{\prime}(s)=A_{h, 0} \bar{W}_{h, n}(s)+C_{h} \partial \hat{w}_{n}(s), \\
& \bar{W}_{h, n}(0)=\Psi_{\frac{k}{2}}^{P_{h} f, t_{n}}\left(P_{h} u\left(t_{n}\right)\right),
\end{aligned}
$$

and $\hat{w}_{n}(s)$ is that in $(27)$.

Then, for the local error $\rho_{h, n+1}=P_{h} u\left(t_{n+1}\right)-\bar{U}_{h, n+1}$, we have the following results:

Theorem 14. Let us assume the same hypotheses of Theorem 7, also that $f \in C^{2}\left([0, T] \times \mathbb{C}^{N}, \mathbb{C}^{N}\right)$, that $\Psi_{k}$ integrates (50) with order $p \geq 2$ and (H1)(H3). Then, when integrating (1) with Strang method as described in (47), (49) and (51), whenever $u$ and $f$ satisfy

$$
u\left(t_{n}\right), A u\left(t_{n}\right), A^{2} u\left(t_{n}\right), f\left(t_{n}, u\left(t_{n}\right)\right), A f\left(t_{n}, u\left(t_{n}\right)\right) \in Z,
$$

for the space $Z$ in (H2) and $\Psi_{\frac{k}{2}}^{f, t_{n}}$ leaves this space invariant, the local error after full discretization satisfies

$$
\rho_{h, n+1}=O\left(k \varepsilon_{h}+k^{2}\right), \quad A_{h, 0}^{-1} \rho_{h, n+1}=O\left(k \eta_{h}+k^{2} \varepsilon_{h}+k^{3}\right),
$$

where $\varepsilon_{h}$ and $\eta_{h}$ are those in (34).

\subsection{Global errors}

From the first result for the local error $\rho_{h, n+1}$ in Theorem 14, a classical argument for the global error gives $e_{h, n}=O\left(k+\varepsilon_{h}\right)$. However, we also have this finer result, which will be very useful for Dirichlet and non-Dirichlet boundary conditions and parabolic problems:

Theorem 15. Let us assume the same hypotheses of Theorem 14, that the bound (45) is satisfied, and

(i) $u \in C^{4}([0, T], X), f \in C^{3}([0, T] \times X, X), u(t) \in D\left(A^{3}\right)$ and $f(t, u(t)) \in$ $D\left(A^{2}\right)$ for all $t \in[0, T]$ and $A^{3} u, A^{2} f(\cdot, u(\cdot)) \in C^{1}([0, T], X)$,

(ii) $\partial f\left(t_{n}, u\left(t_{n}\right)\right)$ is calculated exactly or just approximately from data according to Subsection 3.2,

(iii) the term in $k^{3}$ for the local error when integrating (28) with $\Psi_{k}$ is differentiable with respect to $t_{n}$,

(iv) $u(\cdot), A u(\cdot), A^{2} u(\cdot), f(\cdot, u(\cdot)), A f(\cdot, u(\cdot)) \in C^{1}([0, T], Z)$.

Then, the global error which turns up when integrating (1) through (47), (49) and (51), satisfies

$$
e_{h, n}=O\left(k^{2}+k \varepsilon_{h}+\eta_{h}\right),
$$

where $\varepsilon_{h}$ and $\eta_{h}$ are those in (34). 


\begin{tabular}{|c|c|c|c|}
\hline & $k=5 \times 10^{-4}$ & $k=2.5 \times 10^{-4}$ & $k=1.25 \times 10^{-4}$ \\
\hline$L^{\infty}$-local error & $1.5838 \mathrm{e}-04$ & $4.2830 \mathrm{e}-05$ & $1.1390 \mathrm{e}-05$ \\
\hline Order & & 1.89 & 1.91 \\
\hline$L^{\infty}$-global error & $6.8139 \mathrm{e}-03$ & $3.4035 \mathrm{e}-03$ & $1.7016 \mathrm{e}-03$ \\
\hline Order & & 1.00 & 1.00 \\
\hline
\end{tabular}

Table 1: Local and global error when integrating the one-dimensional problem corresponding to data (54) and (55) with Dirichlet boundary conditions with the suggested modification of Lie-Trotter method

\begin{tabular}{|c|c|c|c|}
\hline & $k=5 \times 10^{-4}$ & $k=2.5 \times 10^{-4}$ & $k=1.25 \times 10^{-4}$ \\
\hline$L^{\infty}$-local error & $2.1797 \mathrm{e}-05$ & $5.5111 \mathrm{e}-06$ & $1.3884 \mathrm{e}-06$ \\
\hline Order & & 1.98 & 1.99 \\
\hline$L^{\infty}$-global error & $4.3261 \mathrm{e}-05$ & $1.1532 \mathrm{e}-05$ & $3.1544 \mathrm{e}-06$ \\
\hline Order & & 1.91 & 1.87 \\
\hline
\end{tabular}

Table 2: Local and global error when integrating the one-dimensional problem corresponding to data (54) and (55) with Dirichlet boundary conditions with the suggested modification of Strang method

\section{Numerical experiments}

In this section we will show, through some examples, that order reduction is completely avoided with the technique suggested here for Lie-Trotter and Strang exponential splitting methods. For the sake of brevity, we have restricted here to finite differences for the space discretization, although collocation-type methods also satisfy hypotheses of Section 5 .

\subsection{One-dimensional problem}

\begin{tabular}{|c|c|c|c|}
\hline$h$ & $k=5 \times 10^{-4}$ & $k=2.5 \times 10^{-4}$ & $k=1.25 \times 10^{-4}$ \\
\hline $4 \times 10^{-3}$ & $8.3736 \mathrm{e}+01$ & $4.0292 \mathrm{e}+01$ & $1.9080 \mathrm{e}+01$ \\
\hline $2 \times 10^{-3}$ & $3.5786 \mathrm{e}+02$ & $1.6983 \mathrm{e}+02$ & $8.2807 \mathrm{e}+01$ \\
\hline $10^{-3}$ & $1.7732 \mathrm{e}+03$ & $7.1818 \mathrm{e}+02$ & $3.4211 \mathrm{e}+02$ \\
\hline
\end{tabular}

Table 3: Local error when integrating the one-dimensional problem corresponding to data (54) and (55) with Dirichlet boundary conditions with the standard implementation of Lie-Trotter method

Firstly, we consider (1) where $X=C([0,1])$ and $A$ is the second-order space derivative. Moreover, we take

$$
u_{0}(x)=e^{x^{3}}, \quad f(t, u)=u^{2}-e^{t+x^{3}}\left(9 x^{4}+6 x+e^{t+x^{3}}-1\right),
$$




\begin{tabular}{|c|c|c|c|}
\hline$h$ & $k=5 \times 10^{-4}$ & $k=2.5 \times 10^{-4}$ & $k=1.25 \times 10^{-4}$ \\
\hline $4 \times 10^{-3}$ & $1.0268 \mathrm{e}+02$ & $4.9388 \mathrm{e}+01$ & $2.3507 \mathrm{e}+01$ \\
\hline $2 \times 10^{-3}$ & $4.4299 \mathrm{e}+02$ & $2.0814 \mathrm{e}+02$ & $1.0131 \mathrm{e}+02$ \\
\hline $10^{-3}$ & $2.3280 \mathrm{e}+03$ & $8.8910 \mathrm{e}+02$ & $4.1921 \mathrm{e}+02$ \\
\hline
\end{tabular}

Table 4: Global error when integrating the one-dimensional problem corresponding to data (54) and (55) with Dirichlet boundary conditions with the standard implementation of LieTrotter method

\begin{tabular}{|c|c|c|c|}
\hline$h$ & $k=5 \times 10^{-4}$ & $k=2.5 \times 10^{-4}$ & $k=1.25 \times 10^{-4}$ \\
\hline $4 \times 10^{-3}$ & $4.0258 \mathrm{e}+01$ & $1.9020 \mathrm{e}+01$ & $8.5800 \mathrm{e}+00$ \\
\hline $2 \times 10^{-3}$ & $1.6985 \mathrm{e}+02$ & $8.2785 \mathrm{e}+01$ & $4.0137 \mathrm{e}+01$ \\
\hline $10^{-3}$ & $7.1836 \mathrm{e}+02$ & $3.4214 \mathrm{e}+02$ & $1.6796 \mathrm{e}+02$ \\
\hline
\end{tabular}

Table 5: Local error when integrating the one-dimensional problem corresponding to data (54) and (55) with Dirichlet boundary conditions with the standard implementation of Strang method

and for the Dirichlet boundary conditions,

$$
g_{0}(t)=e^{t}, \quad g_{1}(t)=e^{t+1},
$$

so that the exact solution of the problem is

$$
u(x, t)=e^{t+x^{3}} .
$$

For the space discretization, we take $h=1 /(N+1)$ and we consider the nodes $x_{j}=j h, j=0, \ldots, N+1$. Then, the discrete space is $\mathbb{C}^{N}$, where $N$ is the number of interior nodes, and the second derivative is approximated by means of the standard second-order difference scheme. Moreover, $P_{h}$ is the projection on the interior nodal values, $A_{h, 0}=\operatorname{tridiag}(1,-2,1) / h^{2}$ and $C_{h} g(t)=\left[g_{0}(t), 0, \ldots, 0, g_{1}(t)\right]^{T} / h^{2}$.

Hypothesis (H1a) can be checked to be satisfied by using the logarithmic norm of matrix $A_{h, 0}$, which is given by [14]

$$
\mu\left(A_{h, 0}\right)=\lim _{\tau \rightarrow 0^{+}} \frac{\left\|I+\tau A_{h, 0}\right\|_{h}-1}{\tau} .
$$

From the logarithmic norm, we obtain the bound

$$
\left\|e^{t A_{h, 0}}\right\|_{h} \leq e^{t \mu\left(A_{h, 0}\right)} .
$$

In particular, with the maximum norm $\left(\|\mathbf{u}\|_{\infty}=\max _{i}\left|u_{i}\right|\right)$, which is the one being used in our examples, we have

$$
\mu_{\infty}(A)=\max _{i}\left(\Re\left(a_{i i}\right)+\sum_{j \neq i}\left|a_{j i}\right|\right) .
$$




\begin{tabular}{|c|c|c|c|}
\hline$h$ & $k=5 \times 10^{-4}$ & $k=2.5 \times 10^{-4}$ & $k=1.25 \times 10^{-4}$ \\
\hline $4 \times 10^{-3}$ & $4.9213 \mathrm{e}+01$ & $2.3259 \mathrm{e}+01$ & $1.0516 \mathrm{e}+01$ \\
\hline $2 \times 10^{-3}$ & $2.0805 \mathrm{e}+02$ & $1.0119 \mathrm{e}+02$ & $4.9048 \mathrm{e}+01$ \\
\hline $10^{-3}$ & $8.8909 \mathrm{e}+02$ & $4.1915 \mathrm{e}+02$ & $2.0530 \mathrm{e}+02$ \\
\hline
\end{tabular}

Table 6: Global error when integrating the one-dimensional problem corresponding to data (54) and (55) with Dirichlet boundary conditions with the standard implementation of Strang method

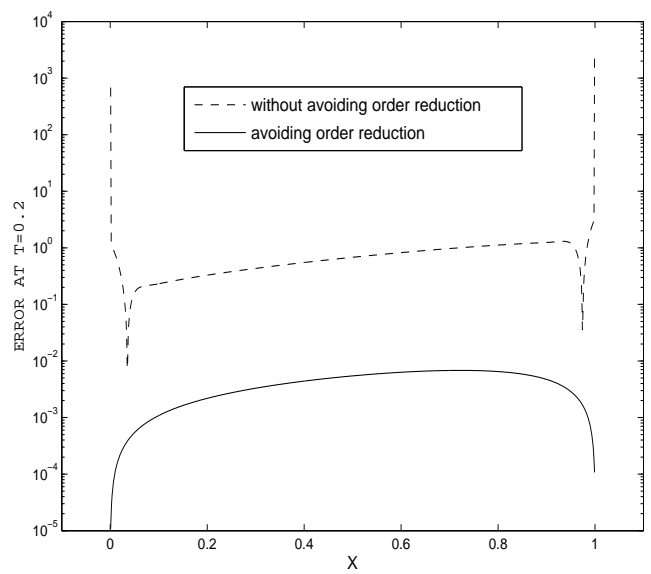

Figure 1: Error at each node at final time when integrating the one-dimensional problem corresponding to data (54) and (55) avoiding and not avoiding order reduction with LieTrotter

For the above $A_{h, 0}$, it is easily seen that $\mu\left(A_{h, 0}\right)_{\infty}=0$ and (H1a) holds. On the other hand, (H1b) can be verified directly from the formula of $A_{h, 0}^{-1}$ and it happens that $\left\|A_{h, 0}^{-1}\right\| \leq \frac{1}{8}$. Moreover, in this case (H2) is true with $Z=$ $C^{4}([0,1]),\|v\|_{Z}=\|v\|_{\infty}+\left\|v_{x x x x}\right\|_{\infty}$ and $\varepsilon_{h}, \eta_{h}=O\left(h^{2}\right)$, a discrete maximum principle applies [26] and $f$ satisfies (H3).

Calculating $\varphi_{j}\left(k A_{h, 0}\right) C_{h} g(t)$ just corresponds to making a linear combination of the first and last column of $\varphi_{j}\left(k A_{h, 0}\right)$, which can be both calculated once and for all at the very beginning for fixed stepsize $k$. As integrator $\Psi_{k}$, we have considered the 4th-order classical Runge-Kutta method.

Considering the technique suggested in this paper, for $h=10^{-3}$, we have obtained the results in Table 1 when integrating till time $T=0.2$ with LieTrotter method and those in Table 2 with Strang method. It is clear that orders 2 and 1 are obtained for the local and global errors respectively when integrating with Lie-Trotter and order 2 for the local and global errors when integrating with Strang method, as assured by Theorems 10, 11, 14 and 15 when 


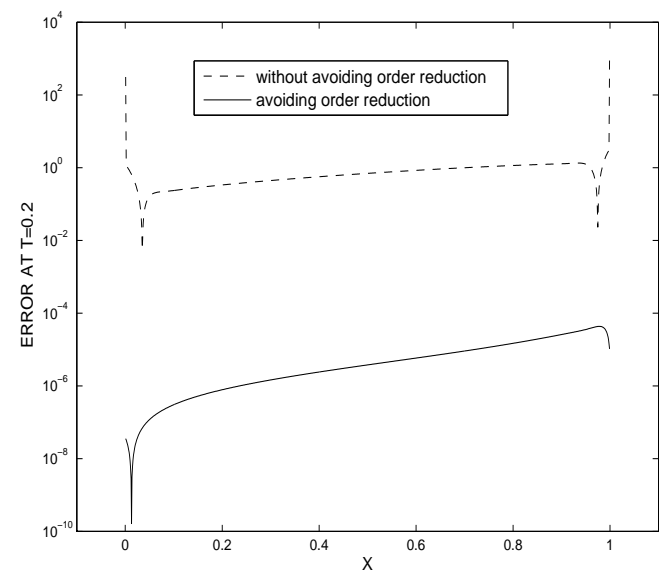

Figure 2: Error at each node at final time when integrating the one-dimensional problem corresponding to data (54) and (55) avoiding and not avoiding order reduction with Strang

the error in space is negligible. (This seems to be the case because decreasing $h$ does not practically change the errors.)

In order to better appreciate the advantage with respect to the standard technique (32), we also show for this problem the results when not avoiding order reduction for both Lie-Trotter and Strang methods for the same values of the parameters $h$ and $k$. In Tables $3,4,5$ and 6 we can observe that, not only the order is just 1 for both local and global error, but also the size of errors is unacceptable and even grows when $h$ diminishes. This is not very surprising to us since that has also been observed when exponential Lawson methods integrate time-dependent boundary value problems in the standard way [3]. On the other hand, we also show the error at each node in space after the final time of integration when avoiding and not avoiding order reduction with $k=5 \times 10^{-4}$. Figures 1 and 2 make it obvious that the main difference in the size of errors comes from the boundary, which is natural taking into account that the technique to avoid order reduction consists of refining the boundary values of the split subproblems.

Let us now consider the same problem as for the previous experiment, but with a Neumann boundary condition at the right boundary. More precisely, the boundary conditions are

$$
\begin{aligned}
u(0, t) & =g_{0}(t), \\
u_{x}(1, t) & =g_{1}(t) .
\end{aligned}
$$

with $g_{0}(t)=e^{t}$ and $g_{1}(t)=3 e^{1+t}$.

In this case, the values in the node $x=1$ are included and the discrete space is $\mathbb{C}^{N+1}$, the matrix $A_{h, 0}$ is the same as in the previous experiment except for 


\begin{tabular}{|c|c|c|c|}
\hline & $k=5 \times 10^{-4}$ & $k=2.5 \times 10^{-4}$ & $k=1.25 \times 10^{-4}$ \\
\hline$L^{\infty}$-local error & $2.0286 \mathrm{e}-04$ & $5.1444 \mathrm{e}-05$ & $1.2795 \mathrm{e}-05$ \\
\hline Order & & 1.98 & 2.01 \\
\hline$L^{\infty}$-global error & $3.9872 \mathrm{e}-02$ & $1.9887 \mathrm{e}-02$ & $9.9237 \mathrm{e}-03$ \\
\hline Order & & 1.00 & 1.00 \\
\hline
\end{tabular}

Table 7: Local and global error when integrating the one-dimensional problem corresponding to data (54), with Dirichlet and Neumann boundary conditions (56), with the suggested modification of Lie-Trotter method

the last row which is $[0, \ldots, 0,2,-2] / h^{2}$ now, and

$$
C_{h} \partial u(t)=\left[g_{0}(t) / h^{2}, 0, \ldots, 0,2 g_{1}(t) / h\right]^{T} .
$$

Again, hypotheses (H1)-(H3) are satisfied but with $\left\|A_{h, 0}^{-1}\right\|_{\infty} \leq 1 / 2$, another discrete maximum principle, $Z=C^{4}([0,1])$ and

$$
\|v\|_{Z}=\|v\|_{\infty}+\max \left(\left\|v_{x x x}\right\|_{\infty},\left\|v_{x x x x}\right\|_{\infty}\right) .
$$

Notice that now, as it is proved by using Taylor expansions, all components of $A_{h, 0}\left(R_{h} u-P_{h} u\right)$ are $O\left(h^{2}\left\|u_{x x x x}\right\|_{\infty}\right)$ except for the last component which just decreases as $O\left(h\left\|u_{x x x}\right\|_{\infty}\right)$. Therefore, $\varepsilon_{h}$ is $O(h)$ and $\eta_{h}$ is, in principle, also $O(h)$ for every $u \in C^{4}([0,1])$. However,

$$
\begin{aligned}
R_{h} u-P_{h} u & =A_{h, 0}^{-1}\left[\begin{array}{c}
O\left(h^{2}\left\|u_{x x x x}\right\|_{\infty}\right) \\
\vdots \\
O\left(h^{2}\left\|u_{x x x x}\right\|_{\infty}\right) \\
0
\end{array}\right]+A_{h, 0}^{-1}\left[\begin{array}{c}
0 \\
\vdots \\
0 \\
O\left(h\left\|u_{x x x}\right\|_{\infty}\right)
\end{array}\right] \\
& =O\left(h^{2}\right)\left\|u_{x x x x}\right\|_{\infty}+O\left(h^{2}\right)\left\|u_{x x x}\right\|_{\infty} A_{h, 0}^{-1}\left[\begin{array}{c}
0 \\
\vdots \\
0 \\
2 / h
\end{array}\right]
\end{aligned}
$$

where, for the last equality, we have used (H1b). Taking now (H2c) into account, $A_{h, 0}^{-1}[0 \ldots 02 / h]^{T}$ is bounded and therefore $\eta_{h}$ is in fact $O\left(h^{2}\right)$.

The results which are obtained with the technique which is proposed in this paper are shown in Table 7 for Lie-Trotter with $h=10^{-3}$ and in Table 8 for Strang with $h=2.5 \times 10^{-4}$. In both cases, the global errors are measured at time $T=0.2$. We see that, for Lie-Trotter, orders 2 and 1 are observed for the local and global error respectively when $k$ decreases, while for Strang, order 2 is obtained for both the local and global errors. These results corroborate Theorems 10, 12, 14 and 15 . 


\begin{tabular}{|c|c|c|c|}
\hline & $k=1 \times 10^{-3}$ & $k=5 \times 10^{-4}$ & $k=2.5 \times 10^{-4}$ \\
\hline$L^{\infty}$-local error & $2.6922 \mathrm{e}-05$ & $5.0772 \mathrm{e}-06$ & $9.1626 \mathrm{e}-07$ \\
\hline Order & & 2.41 & 2.47 \\
\hline$L^{\infty}$-global error & $1.8549 \mathrm{e}-04$ & $4.6220 \mathrm{e}-05$ & $1.0814 \mathrm{e}-05$ \\
\hline Order & & 2.00 & 2.10 \\
\hline
\end{tabular}

Table 8: Local and global error when integrating the one-dimensional problem corresponding to data (54), with Dirichlet and Neumann boundary conditions (56), with the suggested modification of Strang method

\begin{tabular}{|c|c|c|c|}
\hline & $k=5 \times 10^{-3}$ & $k=2.5 \times 10^{-3}$ & $k=1.25 \times 10^{-3}$ \\
\hline$L^{\infty}$-local error & $6.1550 \mathrm{e}-02$ & $1.9049 \mathrm{e}-02$ & $5.7445 \mathrm{e}-03$ \\
\hline Order & & 1.69 & 1.73 \\
\hline$L^{\infty}$-global error & $6.1666 \mathrm{e}-01$ & $2.9307 \mathrm{e}-01$ & $1.4341 \mathrm{e}-01$ \\
\hline Order & & 1.07 & 1.03 \\
\hline
\end{tabular}

Table 9: Local and global error when integrating the two-dimensional problem corresponding to data (57) with the suggested modification of Lie-Trotter method

\subsection{Two-dimensional problem}

We have also considered the two-dimensional problem in the square $\Omega=$ $[0,1] \times[0,1]$ when the operator $A$ is the Laplacian. Moreover, we have considered

$$
\begin{aligned}
& u_{0}(x, y)=e^{x^{3}+y^{3}}, \quad(x, y) \in \Omega, \quad g(t, x, y)=e^{t+x^{3}+y^{3}}, \quad(x, y) \in \partial \Omega \\
& f(t, u, x, y)=u^{2}-e^{t+x^{3}+y^{3}}\left(9\left(x^{4}+y^{4}\right)+6(x+y)+e^{t+x^{3}+y^{3}}-1\right),
\end{aligned}
$$

which has $u(t, x, y)=e^{t+x^{3}+y^{3}}$ as exact solution.

Firstly, for the discretization of the Laplacian we have considered the standard five-point formula [26]. We notice that, in this case, the discrete space is $\mathbb{C}^{N^{2}}$, where $N$ is the number of interior nodes in each direction, and $A_{h, 0}$ is a tridiagonal block-matrix of dimension $N^{2}$. Besides, the matrices in the diagonal are the same and are tridiagonal and the matrices at the subdiagonal and superdiagonal are the same and are diagonal. Notice also that $C_{h} g(t)$ would just have $4 N-4$ non-vanishing components, which is a number which is negligible compared with $N^{2}$, the total number of interior nodes. Again, (H1)-(H3) are satisfied for the infinity norm with $\varepsilon_{h}, \eta_{h}$ being $O\left(h^{2}\right)[26]$.

Tables 9 and 10 show the orders which are observed in time for $h=10^{-2}$ in Table 9 and $h=5 \times 10^{-3}$ in Table 10 when integrating the problem till time $T=1$ with the suggested modifications of Lie-Trotter and Strang method considering again $\Psi_{k}$ as the fourth-order classical Runge-Kutta method. Again, we see that the local and global order for Lie-Trotter are near 2 and 1 respectively and that the local and global order for Strang are near 2.

We also consider the use of a double splitting where we add another splitting in the linear part [1]. For that, we have followed the lines in [1], where the 


\begin{tabular}{|c|c|c|c|}
\hline & $k=1 \times 10^{-2}$ & $k=5 \times 10^{-3}$ & $k=2.5 \times 10^{-3}$ \\
\hline$L^{\infty}$-local error & $5.4278 \mathrm{e}-02$ & $1.6069 \mathrm{e}-02$ & $4.6066 \mathrm{e}-03$ \\
\hline Order & & 1.76 & 1.80 \\
\hline$L^{\infty}$-global error & $3.1796 \mathrm{e}-01$ & $7.7798 \mathrm{e}-02$ & $2.1844 \mathrm{e}-02$ \\
\hline Order & & 2.03 & 1.83 \\
\hline
\end{tabular}

Table 10: Local and global error when integrating the two-dimensional problem corresponding to data (57) with the suggested modification of Strang method

order reduction is avoided in the case of a dimension splitting of the Laplacian operator. We use similar ideas for the discretization of (19) in Lie-Trotter method and that of (26) in Strang method.

More precisely, for the discretization of (19) we firstly consider the problem

$$
\begin{aligned}
z_{n}^{\prime}(s) & =A_{1} z_{n}(s), \\
z_{n}(0) & =u_{n}, \\
\partial_{1} z_{n}(s) & =\partial\left(u\left(t_{n}\right)+s A_{1} u\left(t_{n}\right)\right),
\end{aligned}
$$

with $A_{1} u=\partial_{x x} u$, and $\partial_{1} u=\{u(0, y)=u(1, y), y \in[0,1]\}$. Then,

$$
\begin{aligned}
r_{n}^{\prime}(s) & =A_{2} r_{n}(s) \\
r_{n}(0) & =z_{n}(k), \\
\partial r_{n}(s) & =\partial_{2}\left(u\left(t_{n}\right)+k A_{1} u\left(t_{n}\right)+s A_{2} u\left(t_{n}\right)\right),
\end{aligned}
$$

where $A_{2} u=\partial_{y y} u$ and and $\partial_{2} u=\{u(x, 0)=u(x, 1), x \in[0,1]\}$. Finally, we make $v_{n}(k)=r_{n}(k)$. We apply now the spatial discretization of problems (58) and (59) and we obtain

$$
\begin{aligned}
Z_{h, n}(k)= & e^{k A_{h, 0,1}} U_{h}^{n}+k \varphi_{1}\left(k A_{h, 0,1}\right) C_{h} \partial_{1} u\left(t_{n}\right)+k^{2} \varphi_{2}\left(k A_{h, 0,1}\right) C_{h} \partial_{1} A_{1} u\left(t_{n}\right), \\
R_{h, n}(k)= & e^{k A_{h, 0,2}} Z_{h, n}(k)+k \varphi_{1}\left(k A_{h, 0,2}\right) C_{h} \partial_{2}\left(u\left(t_{n}\right)+k A_{1} u\left(t_{n}\right)\right) \\
& +k^{2} \varphi_{2}\left(k A_{h, 0,2}\right) C_{h} \partial_{2} A_{2} u\left(t_{n}\right),
\end{aligned}
$$

where $A_{h, 0,1}, A_{h, 0,2}, \partial_{1}, \partial_{2}$ are the matrices and the boundaries associated to the spatial discretization of $A_{1}$ and $A_{2}$ respectively.

On the other hand, in order to integrate (26) with Strang method, we firstly consider the problem,

$$
\begin{aligned}
r_{n}^{\prime}(s) & =A_{1} r_{n}(s) \\
r_{n}(0) & =v_{n}\left(\frac{k}{2}\right) \\
\partial_{1} r_{n}(s) & =\partial_{1}\left(u\left(t_{n}\right)+\frac{k}{2} f\left(t_{n}, u\left(t_{n}\right)\right)+s A_{1} u\left(t_{n}\right)\right),
\end{aligned}
$$


then,

$$
\begin{aligned}
\phi_{n}^{\prime}(s) & =A_{2} \phi_{n}(s) \\
\phi_{n}(0) & =r_{n}\left(\frac{k}{2}\right) \\
\partial_{2} \phi_{n}(s) & =\partial_{2}\left(u\left(t_{n}\right)+\frac{k}{2} f\left(t_{n}, u\left(t_{n}\right)\right)+\frac{k}{2} A_{1} u\left(t_{n}\right)+s A_{2} u\left(t_{n}\right)\right),
\end{aligned}
$$

and finally

$$
\begin{aligned}
\mu_{n}^{\prime}(s) & =A_{1} \mu_{n}(s) \\
\mu_{n}(0) & =\phi_{n}(k) \\
\partial_{1} \mu_{n}(s) & =\partial_{1}\left(u\left(t_{n}\right)+\frac{k}{2} f\left(t_{n}, u\left(t_{n}\right)\right)+\frac{k}{2} A_{1} u\left(t_{n}\right)+k A_{2} u\left(t_{n}\right)+s A_{1} u\left(t_{n}\right)\right)
\end{aligned}
$$

and we make $w_{n}(k)=\mu_{n}\left(\frac{k}{2}\right)$. Considering now the spatial discretization of the previous three problems, we obtain

$$
\begin{aligned}
R_{h, n}\left(\frac{k}{2}\right)= & e^{\frac{k}{2} A_{h, 0,1}} V_{h, n}+\frac{k}{2} \varphi_{1}\left(\frac{k}{2} A_{h, 0,1}\right) C_{h} \partial_{1}\left(u\left(t_{n}\right)+\frac{k}{2} f\left(t_{n}, u\left(t_{n}\right)\right)\right) \\
& +\frac{k^{2}}{4} \varphi_{2}\left(\frac{k}{2} A_{h, 0,1}\right) C_{h} \partial_{1} A_{1} u\left(t_{n}\right), \\
\Phi_{h, n}(k)= & e^{k A_{h, 0,2}} R_{h, n}\left(\frac{k}{2}\right)+k \varphi_{1}\left(k A_{h, 0,2}\right) C_{h} \partial_{2}\left(u\left(t_{n}\right)+\frac{k}{2} f\left(t_{n}, u\left(t_{n}\right)\right)+\frac{k}{2} A_{1} u\left(t_{n}\right)\right) \\
& +k^{2} \varphi_{2}\left(k A_{h, 0,2}\right) C_{h} \partial_{2} A_{2} u\left(t_{n}\right), \\
\mu_{h, n}\left(\frac{k}{2}\right)= & e^{\frac{k}{2} A_{h, 0,1}} \Phi_{h, n}(k) \\
& +\frac{k}{2} \varphi_{1}\left(\frac{k}{2} A_{h, 0,1}\right) C_{h} \partial_{1}\left(u\left(t_{n}\right)+\frac{k}{2} f\left(t_{n}, u\left(t_{n}\right)\right)+\frac{k}{2} A_{1} u\left(t_{n}\right)+k A_{2} u\left(t_{n}\right)\right) \\
& +\frac{k^{2}}{4} \varphi_{2}\left(\frac{k}{2} A_{h, 0,1}\right) C_{h} \partial_{1} A_{1} u\left(t_{n}\right), \\
W_{h, n}= & \mu_{h, n}\left(\frac{k}{2}\right) .
\end{aligned}
$$

We have considered the standard second order symmetric finite difference scheme for the discretization of $A_{1}$ and $A_{2}$. Notice that this procedure will be especially efficient since now the matrices $A_{h, 0, j}(j=1,2)$ for space discretization in one or another direction are block-diagonal matrices after reordering and, moreover, the blocks are tridiagonal. Therefore, multiplying $e^{k A_{h, 0, j}}$ or $\varphi_{l}\left(k A_{h, 0, j}\right)$ times a vector of size $N^{2}$ just corresponds to $N$ products of a matrix of dimension $N \times N$ times a vector of size $N$. Moreover, many components of $C_{h} g(t)$ will vanish.

Although we do not make the analysis for this double splitting, it is natural to suspect that order reduction is also being completely avoided. Tables 11 and 12 corroborate that behavior with $h=10^{-2}$ and $h=5 \times 10^{-3}$ respectively. 


\begin{tabular}{|c|c|c|c|}
\hline & $k=5 \times 10^{-3}$ & $k=2.5 \times 10^{-3}$ & $k=1.25 \times 10^{-3}$ \\
\hline$L^{\infty}$-local error & $6.9693 \mathrm{e}-02$ & $1.9980 \mathrm{e}-02$ & $5.8275 \mathrm{e}-03$ \\
\hline Order & & 1.80 & 1.78 \\
\hline$L^{\infty}$-global error & $6.1373 \mathrm{e}-01$ & $2.9240 \mathrm{e}-01$ & $1.4325 \mathrm{e}-01$ \\
\hline Order & & 1.07 & 1.03 \\
\hline
\end{tabular}

Table 11: Local and global error when integrating the two-dimensional problem corresponding to data (57) with the double splitting of Lie-Trotter method and second-order difference scheme in space

\begin{tabular}{|c|c|c|c|}
\hline & $k=10^{-2}$ & $k=5 \times 10^{-3}$ & $k=2.5 \times 10^{-3}$ \\
\hline$L^{\infty}$-local error & $6.6803 \mathrm{e}-02$ & $1.8862 \mathrm{e}-02$ & $5.2890 \mathrm{e}-03$ \\
\hline Order & & 1.82 & 1.83 \\
\hline$L^{\infty}$-global error & $3.5131 \mathrm{e}-01$ & $8.9572 \mathrm{e}-02$ & $2.3855 \mathrm{e}-02$ \\
\hline Order & & 1.97 & 1.91 \\
\hline
\end{tabular}

Table 12: Local and global error when integrating the two-dimensional problem corresponding to data (57) with the double splitting of Strang method and second-order difference scheme in space

\section{Conclusions}

In this paper a technique is suggested to avoid order reduction when integrating reaction-diffusion initial boundary value problems with time-dependent boundary values with Lie-Trotter and Strang splitting methods. We have made a through analysis for the error both in space and time and we have considered not only Dirichlet, but also Neumann and Robin boundary conditions. More precisely, we have specified how to calculate either exactly or approximately the required boundary values for the split subproblems for the different types of boundary conditions and we have even inserted into the analysis the possible error coming from this approximation.

We have numerically verified the great improvement in accuracy with respect to the standard way of implementing the methods which, as it is natural, is more obvious near the boundary.

The results in this paper have already been extended in the set of nonlinear problems to exponential Lawson methods [11] and some research is also being done on its extension to other Runge-Kutta type exponential methods [9].

\section{Acknowledgements}

This work has been supported by Ministerio de Ciencia, Innovación y Universidades, FEDER and Junta de Castilla y León through projects MTM 201566837-P, VA024P17, VA041P17 and VA105G18. 


\section{References}

[1] I. Alonso-Mallo, B. Cano And N. Reguera, Avoiding order reduction when integrating linear initial boundary value problems with exponential splitting methods, IMA J. Numer. Anal., 38 (2018), 1294-1323.

[2] I. Alonso-Mallo, B. Cano And N. Reguera, Analysis of order reduction when integrating linear initial boundary value problems with Lawson methods, Appl. Numer. Math., 118 (2017), 64-74.

[3] I. Alonso-Mallo, B. Cano And N. Reguera, Avoiding order reduction when integrating linear initial boundary value problems with Lawson methods, IMA J. Numer. Anal., 37 (2017), 2091-2119.

[4] I. Alonso-Mallo, B. CAno And N. Reguera, Looking for efficiency when avoiding order reduction in nonlinear problems with Strang splitting, http://arxiv.org/abs/1709.09849.

[5] I. Alonso-Mallo, B. Cano and N. Reguera, Comparison of efficiency among different techniques to avoid order reduction with Strang splitting, submitted for publication.

[6] I. Alonso-Mallo And C. Palencia, On the convolutions operators arising in the study of abstract initial boundary value problems, Proc. Royal Soc. Edinburgh. 126A (1996), 515-539.

[7] W. ARENDT, Semigroups and evolution equations: functional calculus, regularity and kernel estimates. Evolutionary equations. Vol. I, 1-85, Handb. Differ. Equ., North-Holland, Amsterdam, 2004.

[8] W. Arendt, Resolvent positive operators and inhomogeneous boundary conditions, Ann. Scuola Norm. Sup. Pisa Cl Sci. (4), 29 (2000), 639-670.

[9] B. Cano And M. J. Moreta, How to avoid order reduction when RungeKutta exponential methods integrate reaction-diffusion initial boundary value problems, in preparation.

[10] B. Cano And N. Reguera, Avoiding order reduction when integrating nonlinear Schrödinger equation with Strang method, J. Comp. Appl. Math., 316 (2017), 86-99.

[11] B. Cano And N. Reguera, How to avoid order reduction when Lawson methods integrate reaction-diffusion initial boundary value problems, submitted for publication.

[12] H. Cartan, Calcul différentiel. Hermann, Paris, 1967.

[13] J. Connors, J.W. Banks, J.A. Hittinger, And C.S. Woodward, Quantification of errors for operator-split advection-diffusion calculations, Comput. Methods Appl. Mech. Engrg. 272 (2014), 181-197. 
[14] G. DAhlquist, Stability and error bounds in the numerical integration of ordinary differential equations. Trans. Royal Inst. of Technology. No. 130, 1959. Thesis 1958.

[15] L. Einkemmer And A. Ostermann, Overcoming order reduction in diffusion-reaction splitting. Part 1: Dirichlet boundary conditions, SIAM J. Sci. Comput. 37 (3) (2015), A1577-A1592.

[16] L. EInKEMmer AND A. Ostermann, Overcoming order reduction in diffusion-reaction splitting. Part 2: Oblique boundary conditions, SIAM J. Sci. Comput. 38 (2016) A3471-A3757.

[17] E. Faou, A. Ostermann And K. Schratz, Analysis of exponential splitting methods for inhomogeneous parabolic equations, IMA J. Numer. Anal. 35 (1) (2015), 161-178.

[18] T. Gockler And V. Grimm, Convergence analysis of an extended Krylov subspace method for the approximation of operator functions in exponential integrators, SIAM J. Numer. Anal. 51 (4) (2013), 2189-2213.

[19] E. Hansen, F. Kramer, A. Ostermann, A second-order positivity preserving scheme for semilinear parabolic problems, Appl. Numer. Math. 62 (2012), no. 10, 1428-1435.

[20] D. Henry, Geometric Theory of Demilinear Parabolic Problems, Lectures Notes in Mathematics, 840, Springer verlag, New York, 1981.

[21] M. Hochbruck And A. Ostermann, Exponential Runge-Kutta methods for parabolic problems, Appl. Numer. Math. 53 (2005), no. 2-4, 323-339.

[22] M. Hochbruck And A. Ostermann, Exponential integrators, Acta Numerica (2010) 209-286.

[23] R. J. LeVeque And J. Oliger, Numerical methods based on additive splittings for hyperbolic partial differential equations, Math. Comp. 40 (1983), no. $162,469-497$.

[24] C. Palencia And I. Alonso-Mallo, Abstract initial-boundary value problems, Proceedings of the Royal Society of Edinbourgh. Section A- Mathematics. 124, (1994) 879 - 908.

[25] A. PAZY, Semigroups of Linear Operators and Applications to Partial Differential Equations, Series: Applied Mathematical Sciences, Vol. 44, Springer, New York, Berlin, Heidelberg, Tokyo, 1983.

[26] J. C. Strikwerda, Finite Difference Schemes and Partial Differential Equations, (Wadsworth \& Brooks, United States of America, 1989). 


\section{Appendix}

\subsection{Proof of Theorem 5}

Before proving this, let us first study more thoroughly $\bar{v}_{n}(s)$.

Lemma 16. Under hypotheses (A1)-(A5), the solution of (24) is given by

$$
\bar{v}_{n}(s)=u\left(t_{n}\right)+s A u\left(t_{n}\right)+s^{2} \varphi_{2}\left(s A_{0}\right) A^{2} u\left(t_{n}\right) .
$$

where $\varphi_{2}(z)$ is defined in (13).

Proof. Notice that, using (A5),

$$
\begin{aligned}
\bar{v}_{n}^{\prime}(s)-\hat{v}_{n}^{\prime}(s) & =A \bar{v}_{n}(s)-A u\left(t_{n}\right)=A\left(\bar{v}_{n}(s)-\hat{v}_{n}(s)\right)+A\left(\hat{v}_{n}(s)-u\left(t_{n}\right)\right) \\
& =A\left(\bar{v}_{n}(s)-\hat{v}_{n}(s)\right)+s A^{2} u\left(t_{n}\right), \\
\bar{v}_{n}(0)-\hat{v}_{n}(0) & =0, \\
\partial\left(\bar{v}_{n}(s)-\hat{v}_{n}(s)\right) & =0 .
\end{aligned}
$$

Then,

$\bar{v}_{n}(s)=\hat{v}_{n}(s)+\int_{0}^{s} e^{(s-\tau) A_{0}} \tau A^{2} u\left(t_{n}\right) d \tau=u\left(t_{n}\right)+s A u\left(t_{n}\right)+s^{2} \varphi_{2}\left(s A_{0}\right) A^{2} u\left(t_{n}\right)$.

Denoting by $\bar{w}_{n}(s)$ the solution of

$$
\begin{aligned}
& \bar{w}_{n}^{\prime}(s)=f\left(t_{n}+s, \bar{w}_{n}(s)\right) \\
& \bar{w}_{n}(0)=\bar{v}_{n}(k),
\end{aligned}
$$

it happens that, due to (A4) and (A5),

$$
\begin{aligned}
\bar{w}_{n}(k) & =\bar{v}_{n}(k)+k f\left(t_{n}, \bar{v}_{n}(k)\right)+O\left(k^{2}\right) \\
& =u\left(t_{n}\right)+k A u\left(t_{n}\right)+k f\left(t_{n}, u\left(t_{n}\right)\right)+O\left(k^{2}\right)=u\left(t_{n+1}\right)+O\left(k^{2}\right) .
\end{aligned}
$$

Then,

$$
\begin{aligned}
\rho_{n+1} & =\bar{u}_{n+1}-u\left(t_{n+1}\right)=\Psi_{k}^{f, t_{n}}\left(\bar{v}_{n}(k)\right)-u\left(t_{n+1}\right) \\
& =\left[\Psi_{k}^{f, t_{n}}\left(\bar{v}_{n}(k)\right)-\bar{w}_{n}(k)\right]+\left[\bar{w}_{n}(k)-u\left(t_{n+1}\right)\right]=O\left(k^{p+1}\right)+O\left(k^{2}\right)=O\left(k^{2}\right) .
\end{aligned}
$$

\subsection{Proof of Theorem 6}

Denoting by $\bar{w}_{n}(s)$ the solution of (26) starting from $\Psi_{\frac{k}{2}}^{f, t_{n}}\left(u\left(t_{n}\right)\right)$, we have the following result:

Lemma 17. Under hypotheses (A1)-(A6),

$$
\begin{aligned}
\bar{w}_{n}(s)= & u\left(t_{n}\right)+\frac{k}{2} f\left(t_{n}, u\left(t_{n}\right)\right)+s A u\left(t_{n}\right)+e^{s A_{0}}\left(\Psi_{\frac{k}{2}}^{f, t_{n}}\left(u\left(t_{n}\right)\right)-u\left(t_{n}\right)-\frac{k}{2} f\left(t_{n}, u\left(t_{n}\right)\right)\right) \\
& +\frac{k}{2} s \varphi_{1}\left(s A_{0}\right) A f\left(t_{n}, u\left(t_{n}\right)\right)+s^{2} \varphi_{2}\left(s A_{0}\right) A^{2} u\left(t_{n}\right) .
\end{aligned}
$$


Proof. It can be noticed that, because of (A5)-(A6),

$$
\begin{aligned}
\bar{w}_{n}^{\prime}(s)-\hat{w}_{n}^{\prime}(s) & =A \bar{w}_{n}(s)-A u\left(t_{n}\right)=A\left(\bar{w}_{n}(s)-\hat{w}_{n}(s)\right)+A \hat{w}_{n}(s)-A u\left(t_{n}\right) \\
& =A\left(\bar{w}_{n}(s)-\hat{w}_{n}(s)\right)+\frac{k}{2} A f\left(t_{n}, u\left(t_{n}\right)\right)+s A^{2} u\left(t_{n}\right), \\
\bar{w}_{n}(0)-\hat{w}_{n}(0) & =\Psi_{\frac{k}{2}}^{f, t_{n}}\left(u\left(t_{n}\right)\right)-u\left(t_{n}\right)-\frac{k}{2} f\left(t_{n}, u\left(t_{n}\right)\right), \\
\partial\left(\bar{w}_{n}(s)-\hat{w}_{n}(s)\right) & =0 .
\end{aligned}
$$

Then,

$$
\begin{aligned}
\bar{w}_{n}(s)-\hat{w}_{n}(s)= & e^{s A_{0}}\left(\bar{w}_{n}(0)-\hat{w}_{n}(0)\right)+\int_{0}^{s} e^{(s-\tau) A_{0}}\left[\frac{k}{2} A f\left(t_{n}, u\left(t_{n}\right)\right)+\tau A^{2} u\left(t_{n}\right)\right] d \tau \\
= & e^{s A_{0}}\left(\Psi_{\frac{k}{2}}^{f, t_{n}}\left(u\left(t_{n}\right)\right)-u\left(t_{n}\right)-\frac{k}{2} f\left(t_{n}, u\left(t_{n}\right)\right)\right) \\
& +\frac{k}{2} s \varphi_{1}\left(s A_{0}\right) A f\left(t_{n}, u\left(t_{n}\right)\right)+s^{2} \varphi_{2}\left(s A_{0}\right) A^{2} u\left(t_{n}\right) .
\end{aligned}
$$

In order to prove the theorem, notice that, from the hypothesis on $\Psi_{k},(\mathrm{~A} 4)$ and (A5),

$$
\begin{aligned}
\rho_{n+1}= & \bar{u}_{n+1}-u\left(t_{n+1}\right)=\left[\Psi_{\frac{k}{2}}^{f, t_{n}+\frac{k}{2}}\left(\bar{w}_{n}(k)\right)-\bar{z}_{n}\left(\frac{k}{2}\right)\right]+\left[\bar{z}_{n}\left(\frac{k}{2}\right)-u\left(t_{n+1}\right)\right] \\
= & O\left(k^{p+1}\right)+\bar{w}_{n}(k)+\frac{k}{2} f\left(t_{n}+\frac{k}{2}, \bar{w}_{n}(k)\right) \\
& -u\left(t_{n}\right)-k A u\left(t_{n}\right)-k f\left(t_{n}, u\left(t_{n}\right)\right)+O\left(k^{2}\right) \\
= & O\left(k^{2}\right)
\end{aligned}
$$

where the last equality is deduced from Lemma 17, (A4)-(A6) and the fact that $p \geq 1$.

\subsection{Proof of Theorem 7}

Under the new assumptions, by explicitly writing the term in $k^{2}$ in (63), we have

$$
\begin{aligned}
\rho_{n+1}= & \frac{k^{2}}{4} f_{t}\left(t_{n}, u\left(t_{n}\right)\right)+\frac{k^{2}}{4} f_{u}\left(t_{n}, u\left(t_{n}\right)\right) f\left(t_{n}, u\left(t_{n}\right)\right)+\frac{k^{2}}{2} f_{u}\left(t_{n}, u\left(t_{n}\right)\right) A u\left(t_{n}\right) \\
& +\frac{k^{2}}{8}\left[f_{t}\left(t_{n}, u\left(t_{n}\right)\right)+f_{u}\left(t_{n}, u\left(t_{n}\right)\right) f\left(t_{n}, u\left(t_{n}\right)\right)\right] \\
& +\frac{k^{2}}{8} e^{k A_{0}}\left[f_{t}\left(t_{n}, u\left(t_{n}\right)\right)+f_{u}\left(t_{n}, u\left(t_{n}\right)\right) f\left(t_{n}, u\left(t_{n}\right)\right)\right] \\
& +\frac{k^{2}}{2} \varphi_{1}\left(k A_{0}\right) A f\left(t_{n}, u\left(t_{n}\right)\right)+k^{2} \varphi_{2}\left(k A_{0}\right) A^{2} u\left(t_{n}\right)-\frac{k^{2}}{2} u^{\prime \prime}\left(t_{n}\right)+O\left(k^{3}\right) .
\end{aligned}
$$


By applying now $A_{0}^{-1}$, considering (14) and simplifying terms and the notation for the sake of brevity,

$$
\begin{aligned}
A_{0}^{-1} \rho_{n+1}= & k^{2} A_{0}^{-1}\left[\frac{3}{8} f_{t}+\frac{3}{8} f_{u} f+\frac{1}{2} f_{u} A u-\frac{1}{2} u^{\prime \prime}\right]+\frac{k^{2}}{8}\left(k \varphi_{1}\left(k A_{0}\right)+A_{0}^{-1}\right)\left(f_{t}+f_{u} f\right) \\
& +\frac{k^{2}}{2}\left(k \varphi_{2}\left(k A_{0}\right)+A_{0}^{-1}\right) A f+k^{2}\left(k \varphi_{3}\left(k A_{0}\right)+\frac{1}{2} A_{0}^{-1}\right) A^{2} u+O\left(k^{3}\right) \\
= & k^{2} A_{0}^{-1}\left[\frac{1}{2}\left(f_{t}+f_{u} f\right)+\frac{1}{2} f_{u} A u+\frac{1}{2} A f+\frac{1}{2} A^{2} u-\frac{1}{2} u^{\prime \prime}\right]+O\left(k^{3}\right),
\end{aligned}
$$

where, in order to see that the term in bracket vanishes, it suffices to differentiate (1) once with respect to time.

\subsection{Proof of Theorem 10}

Notice that, because of the assumption on $\Psi_{k}$,

$$
\bar{U}_{h}^{n+1}=\Psi_{k}^{f, t_{n}}\left(\bar{V}_{h, n}(k)\right)=\bar{V}_{h, n}(k)+k f\left(t_{n}, \bar{V}_{h, n}(k)\right)+O\left(k^{2}\right) .
$$

On the other hand, making the difference between (42) and (24) multiplied by $P_{h}$,

$$
\begin{aligned}
& \bar{V}_{h, n}^{\prime}(s)-P_{h} \bar{v}_{n}^{\prime}(s)=A_{h, 0}\left(\bar{V}_{h, n}(s)-P_{h} \bar{v}_{n}(s)\right)+A_{h, 0}\left(P_{h}-R_{h}\right) \bar{v}_{n}(s), \\
& \bar{V}_{h, n}(0)-P_{h} \bar{v}_{n}(0)=0 .
\end{aligned}
$$

Then,

$$
\begin{aligned}
\bar{V}_{h, n}(k) & =P_{h} \bar{v}_{n}(k)+\int_{0}^{k} e^{(k-s) A_{h, 0}} A_{h, 0}\left(P_{h}-R_{h}\right) \bar{v}_{n}(s) d s \\
& =P_{h} u\left(t_{n}\right)+k P_{h} A u\left(t_{n}\right)+O\left(k^{2}\right)+O\left(k \varepsilon_{h}\right),
\end{aligned}
$$

where the last equality comes from Lemma 16, (H1) and (H2). From the definition of $\rho_{h, n}$, and again using the accuracy of the numerical integrator $\Psi_{k}$,

$$
\begin{aligned}
\rho_{h, n+1} & =P_{h} u\left(t_{n+1}\right)-\bar{U}_{h, n+1}=P_{h}\left(u\left(t_{n+1}\right)-\bar{u}_{n+1}\right)+\left(P_{h} \bar{u}_{n+1}-\bar{U}_{h, n+1}\right) \\
& =P_{h} \rho_{n+1}+P_{h} \bar{u}_{n+1}-\bar{V}_{h, n}(k)-k f\left(t_{n}, \bar{V}_{h, n}(k)\right)+O\left(k^{2}\right) .
\end{aligned}
$$

Considering now Theorem 5, (30), (64), (H3) and the fact that, because of Lemma 16,

$$
\begin{aligned}
\bar{u}_{n+1} & =\Psi_{k}^{f, t_{n}}\left(\bar{v}_{n}(k)\right)=\bar{v}_{n}(k)+k f\left(t_{n}, \bar{v}_{n}(k)\right)+O\left(k^{2}\right) \\
& =u\left(t_{n}\right)+k A u\left(t_{n}\right)+k f\left(t_{n}, u\left(t_{n}\right)\right)+O\left(k^{2}\right),
\end{aligned}
$$

the first part of the theorem is proved. To prove the second bound in (44), it suffices to apply the uniformly bounded matrix $A_{h, 0}^{-1}$ to the above formulas and to take the second part of (34) into account. 


\subsection{Proof of Theorem 11}

It suffices to notice that

$$
\begin{aligned}
e_{h, n+1} & =\left[P_{h} u\left(t_{n+1}\right)-\bar{U}_{h}^{n+1}\right]+\left[\bar{U}_{h}^{n+1}-U_{h}^{n+1}\right] \\
& =\rho_{h, n+1}+\Psi_{k}^{f, t_{n}}\left(\bar{V}_{h, n}(k)\right)-\Psi_{k}^{f, t_{n}}\left(V_{h, n}(k)\right) \\
& =\rho_{h, n+1}+\bar{W}_{h, n}(k)-W_{h, n}(k)+O\left(k^{p+1}\right),
\end{aligned}
$$

where $\bar{W}_{h, n}(k)$ is the solution of (37) with initial condition $\bar{V}_{h, n}(k)$, and the definition of $\rho_{h, n+1},(39)$ and (41) have been used. Then, considering (37),

$$
\begin{aligned}
\bar{W}_{h, n}^{\prime}(t)-W_{h, n}^{\prime}(s) & =f\left(t_{n}+s, \bar{W}_{h, n}(s)\right)-f\left(t_{n}+s, W_{h, n}(s)\right), \\
\bar{W}_{h, n}(0)-W_{h, n}(0) & =\bar{V}_{h, n}(k)-V_{h, n}(k),
\end{aligned}
$$

and

$$
\begin{aligned}
& \bar{W}_{h, n}(t)-W_{h, n}(t) \\
& \quad=\bar{W}_{h, n}(0)-W_{h, n}(0)+\int_{0}^{t}\left[f\left(t_{n}+s, \bar{W}_{h, n}(s)\right)-f\left(t_{n}+s, W_{h, n}(s)\right)\right] d s \\
& \quad=\bar{V}_{h, n}(k)-V_{h, n}(k)+\int_{0}^{t}\left[f\left(t_{n}+s, \bar{W}_{h, n}(s)\right)-f\left(t_{n}+s, W_{h, n}(s)\right)\right] d s .
\end{aligned}
$$

Taking norms,

$$
\begin{aligned}
& \left\|\bar{W}_{h, n}(t)-W_{h, n}(t)\right\|_{h} \\
& \quad \leq\left\|\bar{V}_{h, n}(k)-V_{h, n}(k)\right\|_{h}+\int_{0}^{t}\left\|f\left(t_{n}+s, \bar{W}_{h, n}(s)\right)-f\left(t_{n}+s, W_{h, n}(s)\right)\right\|_{h} d s \\
& \quad \leq\left\|\bar{V}_{h, n}(k)-V_{h, n}(k)\right\|_{h}+\int_{0}^{t} L\left\|\bar{W}_{h, n}(s)-W_{h, n}(s)\right\|_{h} d s
\end{aligned}
$$

where (H3) has been used. We can then apply Gronwall lemma and deduce that

$$
\left\|\bar{W}_{h, n}(t)-W_{h, n}(t)\right\|_{h} \leq e^{L t}\left\|\bar{V}_{h, n}(k)-V_{h, n}(k)\right\|_{h} .
$$

Moreover,

$$
\begin{aligned}
& \bar{W}_{h, n}(k)-W_{h, n}(k) \\
& \quad=\bar{V}_{h, n}(k)-V_{h, n}(k)+\int_{0}^{k}\left[f\left(t_{n}+s, \bar{W}_{h, n}(s)\right)-f\left(t_{n}+s, W_{h, n}(s)\right)\right] d s \\
& \quad=\bar{V}_{h, n}(k)-V_{h, n}(k)+E\left(\bar{V}_{h, n}(k), V_{h, n}(k)\right),
\end{aligned}
$$

where

$\left\|E\left(\bar{V}_{h, n}(k), V_{h, n}(k)\right)\right\|_{h} \leq L \int_{0}^{k} e^{s L}\left\|\bar{V}_{h, n}(k)-V_{h, n}(k)\right\|_{h} d s \leq k C\left\|\bar{V}_{h, n}(k)-V_{h, n}(k)\right\|_{h}$, for a constant $C$ which is independent of $k$, when $k$ is small enough. 
On the other hand, by making the difference between (36) and (42),

$$
\begin{aligned}
& \bar{V}_{h, n}^{\prime}(s)-V_{h, n}^{\prime}(s)=A_{h, 0}\left(\bar{V}_{h, n}(s)-V_{h, n}(s)\right), \\
& \bar{V}_{h, n}(0)-V_{h, n}(0)=P_{h} u\left(t_{n}\right)-U_{h}^{n},
\end{aligned}
$$

from what

$$
\bar{V}_{h, n}(k)-V_{h, n}(k)=e^{k A_{h, 0}}\left(P_{h} u\left(t_{n}\right)-U_{h}^{n}\right),
$$

and then

$$
e_{h, n+1}=e^{k A_{h, 0}} e_{h, n}+\rho_{h, n+1}+k \bar{E}_{h}\left(U_{h}^{n}, P_{h} u\left(t_{n}\right)\right),
$$

where, for some constant $\bar{C}$,

$$
\left\|\bar{E}_{h}\left(U_{h}^{n}, P_{h} u\left(t_{n}\right)\right)\right\|_{h} \leq \bar{C}\left\|e_{h, n}\right\|_{h} .
$$

From here,

$$
\begin{aligned}
e_{h, n}= & e^{n k A_{h, 0}} e_{h, 0}+\sum_{l=1}^{n} e^{(n-l) k A_{h, 0}} \rho_{h, l} \\
& +k \sum_{l=0}^{n-1} e^{(n-l-1) k A_{h, 0}} \bar{E}_{h}\left(U_{h}^{l}, P_{h} u\left(t_{l}\right)\right) .
\end{aligned}
$$

As $e_{h, 0}=P_{h} u(0)-U_{h}^{0}=0$, by taking norms,

$$
\left\|e_{h, n}\right\|_{h} \leq O\left(k+\varepsilon_{h}\right)+k \overline{\bar{C}} \sum_{l=0}^{n-1}\left\|e_{h, l}\right\|_{h},
$$

and using the discrete Gronwall lemma, the result follows.

\subsection{Proof of Theorem 12}

The proof is very similar to that of Theorem 11 with the difference that now (67) must be substituted by

$\bar{V}_{h, n}^{\prime}(s)-V_{h, n}^{\prime}(s)=A_{h, 0}\left(\bar{V}_{h, n}(s)-V_{h, n}(s)\right)-s\left[C_{h} \partial f\left(t_{n}, u\left(t_{n}\right)\right)-C_{h, n}^{*}\left(U_{h}^{n}\right)\right]$, where $C_{h, n}^{*}\left(U_{h}^{n}\right)$ corresponds to approximating $C_{h} \partial f\left(t_{n}, u\left(t_{n}\right)\right)$ according to Subsection 3.2. Therefore,

$$
\begin{aligned}
& \bar{V}_{h, n}(k)-V_{h, n}(k) \\
& \quad=e^{k A_{h, 0}\left(P_{h} u\left(t_{n}\right)-U_{n}^{n}\right)-k^{2} \varphi_{2}\left(k A_{h, 0}\right)\left[C_{h} \partial f\left(t_{n}, u\left(t_{n}\right)\right)-C_{h, n}^{*}\left(U_{h}^{n}\right)\right]} \\
& \quad=e^{k A_{h, 0}}\left(P_{h} u\left(t_{n}\right)-U_{h}^{n}\right)-k\left[\varphi_{1}\left(k A_{h, 0}\right)-I\right] A_{h, 0}^{-1}\left[C_{h} \partial f\left(t_{n}, u\left(t_{n}\right)\right)-C_{h, n}^{*}\left(U_{h}^{n}\right)\right] \\
& \quad=e^{k A_{h, 0}}\left(P_{h} u\left(t_{n}\right)-U_{n}^{n}\right)+O\left(k\left\|e_{h, n}\right\|_{h}\right),
\end{aligned}
$$


where the definition of $\varphi_{1}$ in (13) has been considered as well as (14), (H2c) and the fact that $\phi \in C^{2}(\mathbb{C}, \mathbb{C})$. From this, (69) still applies for some other function $\bar{E}_{h}$ also satisfying (68). Then, we write one of the terms in (69) as

$$
\begin{aligned}
& \sum_{l=1}^{n} e^{k(n-l) A_{h, 0}} \rho_{h, l} \\
& \quad=\left(\sum_{r=1}^{n-1} e^{r k A_{h, 0}}\right) \rho_{h, 1}+\sum_{j=2}^{n-1}\left(\sum_{r=1}^{j-1} e^{r k A_{h, 0}}\right)\left(\rho_{h, n-j+1}-\rho_{h, n-j}\right)+\rho_{h, n} .
\end{aligned}
$$

As the first term in this decomposition can be written as

$$
\left(\sum_{r=1}^{n-1} e^{r k A_{h, 0}}\right) \rho_{h, 1}=\left(k A_{h, 0} \sum_{r=1}^{n-1} e^{r k A_{h, 0}}\right) \frac{1}{k} A_{h, 0}^{-1} \rho_{h, 1},
$$

applying (45) and Theorem 10, this term is proved to be $O\left(k+\eta_{h}\right)$. As for the second term in (70), because of (A4) and (A5), the term in $k^{2}$ in $A_{0}^{-1} \rho_{n+1}$ is differentiable with respect to time $t_{n}$ and therefore, $A_{0}^{-1}\left(\rho_{n-j+1}-\rho_{n-j}\right)=O\left(k^{3}\right)$. When this is used in the local error for the full discretization, $A_{h, 0}^{-1}\left(\rho_{h, n-j+1}-\right.$ $\left.\rho_{h, n-j}\right)=O\left(k^{3}+k^{2} \eta_{h}\right)$, from what

$$
\begin{aligned}
\sum_{j=2}^{n-1}\left(\sum_{r=1}^{j-1} e^{r k A_{h, 0}}\right)\left(\rho_{h, n-j+1}-\rho_{h, n-j}\right) & =\sum_{j=2}^{n-1}\left(k A_{h, 0} \sum_{r=1}^{j-1} e^{r k A_{h, 0}}\right) \frac{1}{k} A_{h, 0}^{-1}\left(\rho_{h, n-j+1}-\rho_{h, n-j}\right) \\
& =O\left(k+\eta_{h}\right) .
\end{aligned}
$$

Finally, using Theorem 10 for the last term in (70), it is clear that

$$
\sum_{l=1}^{n} e^{k(n-l) A_{h, 0}} \rho_{h, l}=O\left(k+\eta_{h}+k \varepsilon_{h}\right),
$$

and the proof of the theorem follows by applying discrete Gronwall lemma to

$$
\left\|e_{h, n}\right\|_{h} \leq O\left(k+\eta_{h}+k \varepsilon_{h}\right)+k \overline{\bar{C}} \sum_{l=0}^{n-1}\left\|e_{h, l}\right\|_{h} .
$$

\subsection{Proof of Theorem 14}

Notice that

$$
\begin{aligned}
\rho_{h, n+1}= & P_{h} u\left(t_{n}\right)-\bar{U}_{h, n+1}=P_{h} \rho_{n+1}+P_{h} \bar{u}_{n+1}-\bar{U}_{h, n+1} \\
= & P_{h} \rho_{n+1}+P_{h} \Psi_{\frac{k}{2}}^{f, t_{n}+\frac{k}{2}}\left(\bar{w}_{n}(k)\right)-\Psi_{\frac{k}{2}}^{f, t_{n}+\frac{k}{2}}\left(\bar{W}_{h, n}(k)\right) \\
= & P_{h} \rho_{n+1}+P_{h} \bar{w}_{n}(k)-\bar{W}_{h, n}(k) \\
& +\frac{k}{2} f\left(t_{n}+\frac{k}{2}, P_{h} \bar{w}_{n}(k)\right)-\frac{k}{2} f\left(t_{n}+\frac{k}{2}, \bar{W}_{h, n}(k)\right) \\
& +\frac{k^{2}}{8}\left[\left(f_{t}+f_{u} f\right)\left(t_{n}+\frac{k}{2}, P_{h} \bar{w}_{n}(k)\right)-\left(f_{t}+f_{u} f\right)\left(t_{n}+\frac{k}{2}, \bar{W}_{h, n}(k)\right)\right] \\
& +O\left(k^{3}\right),
\end{aligned}
$$


where we have used (31), the fact that $\Psi_{\frac{k}{2}}^{f, t_{n}}$ integrates (50) with order $p \geq 2$ and that $f \in C^{2}\left([0, T] \times \mathbb{C}^{N}, \mathbb{C}^{N}\right)$. Now, from (52) and the definition of $\bar{w}_{n}(s)$ in Subsection 4.2,

$$
\begin{aligned}
P_{h} \bar{w}_{n}^{\prime}(s)-\bar{W}_{h, n}^{\prime}(s) & =P_{h} A \bar{w}_{n}(s)-A_{h, 0} \bar{W}_{h, n}(s)-C_{h} \partial \hat{w}_{n}(s) \\
& =A_{h, 0}\left(P_{h} \bar{w}_{n}(s)-\bar{W}_{h, n}(s)\right)+A_{h, 0}\left(R_{h}-P_{h}\right) \bar{w}_{n}(s),
\end{aligned}
$$

where the last term is $O\left(\varepsilon_{h}\right)$ according to Lemma 17, (53) and (H2). Moreover, again by (31),

$$
P_{h} \bar{w}_{n}(0)-\bar{W}_{h, n}(0)=P_{h} \Psi_{\frac{k}{2}}^{f, t_{n}}\left(u\left(t_{n}\right)\right)-\Psi_{\frac{k}{2}}^{f, t_{n}}\left(P_{h} u\left(t_{n}\right)\right)=0 .
$$

Then,

$$
P_{h} \bar{w}_{n}(k)-\bar{W}_{h, n}(k)=\int_{0}^{k} e^{(k-s) A_{h, 0}} A_{h, 0}\left(R_{h}-P_{h}\right) \bar{w}_{n}(s) d s=O\left(k \varepsilon_{h}\right),
$$

and the first result follows by using Theorem 6 .

For the second result, applying $A_{h, 0}^{-1}$ to (72) and using the second formula in (H2b), it follows in the same way that

$$
A_{h, 0}^{-1} \rho_{h, n+1}=A_{h, 0}^{-1} P_{h} \rho_{n+1}+O\left(k \eta_{h}\right)+O\left(k^{2} \varepsilon_{h}\right)+O\left(k^{3}\right) .
$$

Now, the key of the proof is that, if $\omega_{n+1}=A_{0}^{-1} \rho_{n+1}, R_{h} \omega_{n+1}=A_{h, 0}^{-1} P_{h} \rho_{n+1}$. This comes from the fact that $\omega_{n+1}$ is the solution of

$$
A \omega_{n+1}=\rho_{n+1}, \quad \partial \omega_{n+1}=0,
$$

from what $A_{h, 0} R_{h} \omega_{n+1}=P_{h} \rho_{n+1}$. Moreover, we will take into account that

$$
R_{h} \omega_{n+1}=P_{h} \omega_{n+1}+\left(R_{h}-P_{h}\right) \omega_{n+1}=O\left(k^{3}\right)+O\left(k \eta_{h}\right),
$$

where we have used Theorem 7 for the bound of the first term. As for the second, we have used the definition of $\rho_{n+1},(53)$, Lemma 17, hypothesis (H2a) and the fact that $\Psi_{\frac{k}{2}}^{f, t_{n}}$ leaves $Z$ invariant. Because of this, $\omega_{n+1} \in Z$ and $\left\|\omega_{n+1}\right\|_{Z}=O(k)$, and using (H2b) the result follows.

\subsection{Proof of Theorem 15}

Notice that

$$
\begin{aligned}
e_{h, n+1}= & {\left[P_{h} u\left(t_{n+1}\right)-\bar{U}_{h}^{n+1}\right]+\left[\bar{U}_{h}^{n+1}-U_{h}^{n+1}\right] } \\
= & \rho_{h, n+1}+\Psi_{\frac{k}{2}}^{f, t_{n}+\frac{k}{2}}\left(\bar{W}_{h, n}(k)\right)-\Psi_{\frac{k}{2}}^{f, t_{n}+\frac{k}{2}}\left(W_{h, n}(k)\right) \\
= & \rho_{h, n+1}+\bar{W}_{h, n}(k)-W_{h, n}(k)+k E\left(\bar{W}_{h, n}(k), W_{h, n}(k), k\right) \\
& +O\left(k^{p+1}\right),
\end{aligned}
$$


where, for some constant $C$,

$$
\left.\left\|E\left(\bar{W}_{h, n}(k), W_{h, n}(k), k\right)\right\|_{h} \leq C \| \bar{W}_{h, n}(k)-W_{h, n}(k)\right) \|_{h} .
$$

Now, notice that

$\bar{W}_{h, n}^{\prime}(s)-W_{h, n}^{\prime}(s)=A_{h, 0}\left(\bar{W}_{h, n}(s)-W_{h, n}(s)\right)+\left(\frac{k}{2}-s\right)\left[C_{h} \partial f\left(t, u\left(t_{n}\right)\right)-C_{h, n}^{*}\left(U_{h}^{n}\right)\right]$,

$\bar{W}_{h, n}(0)-W_{h, n}(0)=\Psi_{\frac{k}{2}}^{f, t_{n}}\left(P_{h} u\left(t_{n}\right)\right)-\Psi_{\frac{k}{2}}^{f, t_{n}}\left(U_{h}^{n}\right)$,

where $C_{h, n}^{*}\left(U_{h}^{n}\right)$ is the approximation to $\partial f\left(t_{n}, u\left(t_{n}\right)\right)$ calculated according to Subsection 3.2. Then,

$$
\begin{aligned}
& \bar{W}_{h, n}(k)-W_{h, n}(k) \\
& =e^{k A_{h, 0}}\left(\Psi_{\frac{k}{2}}^{f, t_{n}}\left(P_{h} u\left(t_{n}\right)\right)-\Psi_{\frac{k}{2}}^{f, t_{n}}\left(U_{h}^{n}\right)\right) \\
& +k^{2}\left(\frac{1}{2} \varphi_{1}\left(k A_{h, 0}\right)-\varphi_{2}\left(k A_{h, 0}\right)\right)\left[C_{h} \partial f\left(t_{n}, u\left(t_{n}\right)\right)-C_{h, n}^{*}\left(U_{h}^{n}\right)\right] \\
& =e^{k A_{h, 0}}\left(\bar{V}_{h, n}\left(\frac{k}{2}\right)-V_{h, n}\left(\frac{k}{2}\right)+O\left(k^{p+1}\right)\right) \\
& +k\left(\frac{1}{2}\left(e^{k A_{h, 0}}+I\right)-\varphi_{1}\left(k A_{h, 0}\right)\right) A_{h, 0}^{-1}\left[C_{h} \partial f\left(t_{n}, u\left(t_{n}\right)\right)-C_{h, n}^{*}\left(U_{h}^{n}\right)\right] \\
& =e^{k A_{h, 0}}\left(P_{h} u\left(t_{n}\right)-U_{h}^{n}+k E\left(P_{h} u\left(t_{n}\right), U_{h}^{n}, k\right)+O\left(k^{p+1}\right)\right) \\
& +k\left(\frac{1}{2}\left(e^{k A_{h, 0}}+I\right)-\varphi_{1}\left(k A_{h, 0}\right)\right) A_{h, 0}^{-1}\left[C_{h} \partial f\left(t_{n}, u\left(t_{n}\right)\right)-C_{h, n}^{*}\left(U_{h}^{n}\right)\right] \\
& =e^{k A_{h, 0}} e_{h, n}+k \bar{E}\left(P_{h} u\left(t_{n}\right), U_{h}^{n}, k\right)+O\left(k^{p+1}\right),
\end{aligned}
$$

where $\bar{V}_{h, n}(s)$ is the solution of (46) starting at $P_{h} u\left(t_{n}\right)$ and where the definition of $\varphi_{1}$ and $\varphi_{2}$ (13) has been considered as well as (14), (H2c) and the fact that $\Psi_{k}$ integrates (28) with order $p$. Moreover, for some constant $\bar{C}$,

$$
\left\|\bar{E}\left(P_{h} u\left(t_{n}\right), U_{h}^{n}, k\right)\right\|_{h} \leq \bar{C}\left\|e_{h, n}\right\|_{h} .
$$

Then, inserting this in (73),

$$
e_{h, n+1}=e^{k A_{h, 0}} e_{h, n}+k \overline{\bar{E}}\left(P_{h} u\left(t_{n}\right), U_{h}^{n}, k\right)+\rho_{h, n+1}+O\left(k^{p+1}\right),
$$

where, for some constant $\overline{\bar{C}}$,

$$
\left\|\overline{\bar{E}}\left(P_{h} u\left(t_{n}\right), U_{h}^{n}, k\right)\right\|_{h} \leq \overline{\bar{C}}\left\|e_{h, n}\right\|_{h} .
$$

Inductively, this means that

$$
\begin{aligned}
e_{h, n}= & e^{n k A_{h, 0}} e_{h, 0}+\sum_{l=1}^{n} e^{(n-l) k A_{h, 0}}\left(\rho_{h, l}+O\left(k^{p+1}\right)\right) \\
& +k \sum_{l=0}^{n-1} e^{(n-l-1) k A_{h, 0}} \overline{\bar{E}}\left(P_{h} u\left(t_{n}\right), U_{h}^{l}, k\right) .
\end{aligned}
$$


Then, we write one of the terms in (76) as in (70). As the first term in this decomposition can be written as (71), applying (45) and Theorem 14, this term is proved to be $O\left(k^{2}+\eta_{h}\right)$. As for the second term in (70), hypothesis (i) makes that the term in $k^{3}$ in $A_{0}^{-1} \rho_{n+1}$ is differentiable with respect to time $t_{n}$ and therefore, $A_{0}^{-1}\left(\rho_{n-j+1}-\rho_{n-j}\right)=O\left(k^{4}\right)$. When this is used in the local error for the full discretization, $A_{h, 0}^{-1}\left(\rho_{h, n-j+1}-\rho_{h, n-j}\right)=O\left(k^{4}+k^{2} \eta_{h}\right)$, from what

$$
\begin{aligned}
& \sum_{j=2}^{n-1}\left(\sum_{r=1}^{j-1} e^{r k A_{h, 0}}\right)\left(\rho_{h, n-j+1}-\rho_{h, n-j}\right) \\
& \quad=\sum_{j=2}^{n-1}\left(k A_{h, 0} \sum_{r=1}^{j-1} e^{r k A_{h, 0}}\right) \frac{1}{k} A_{h, 0}^{-1}\left(\rho_{h, n-j+1}-\rho_{h, n-j}\right)=O\left(k^{2}+\eta_{h}\right) .
\end{aligned}
$$

Finally, using Theorem 14 for the last term in (70), it is clear that

$$
\sum_{l=1}^{n} e^{i k(n-l) A_{h, 0}} \rho_{h, l}=O\left(k^{2}+k \varepsilon_{h}+\eta_{h}\right) .
$$

By taking then norms and considering that $e_{h, 0}=0$, we have that

$$
\left\|e_{h, n}\right\|_{h} \leq O\left(k^{2}+k \varepsilon_{h}+\eta_{h}\right)+k \overline{\bar{C}} \sum_{l=0}^{n-1}\left\|e_{h, l}\right\|_{h} .
$$

Applying a discrete Gronwall lemma, the result follows. 